\title{
Activity-dependent alteration of early myelin ensheathment in a developing sensory circuit
}

\author{
Zahraa Chorghay $^{1}$ | David MacFarquhar ${ }^{2,3}$ | Vanessa J. Li ${ }^{1}$ | Sarah Aufmkolk ${ }^{1,2,3}$ \\ Anne Schohl $^{1}$ | Paul W. Wiseman ${ }^{2,3}$ | Ragnhildur Thóra Káradóttir ${ }^{4,5}$ | \\ Edward S. Ruthazer ${ }^{1}$ (i)
}

${ }^{1}$ Montreal Neurological Institute-Hospital and Department of Neurology and Neurosurgery, McGill University, Montréal, QC, Canada

2 Department of Chemistry, Otto Maass Building, McGill University, Montréal, QC Canada

${ }^{3}$ Department of Physics, Otto Maass Building, McGill University, Montréal, QC, Canada

${ }^{4}$ Wellcome Trust-Medical Research Council

Cambridge Stem Cell Institute and

Department of Veterinary Medicine,

University of Cambridge, Cambridge, UK

${ }^{5}$ Department of Physiology, BioMedical

Center, Faculty of Medicine, University of

Iceland, Reykjavik, Iceland

\section{Correspondence}

Edward S. Ruthazer, Montreal Neurological Institute-Hospital and Department of Neurology and Neurosurgery, McGill University, 3801 Rue University, Montréal, QC H3A2B4, Canada.

Email:edward.ruthazer@mcgill.ca

Funding information

Fonds de recherche du Québec - Santé, Grant/Award Number: 31036; Montreal Neurological Institute - University of Cambridge Collaboration; European Union's Horizon 2020 Research and Innovation Programme, Grant/Award Number: 771411; Natural Sciences and Engineering Research Council of Canada; Wellcome Trust, Grant/Award Number: 203151/Z/16/Z; UKRI Medical Research Council, Grant/Award Number: MC_PC_17230

\begin{abstract}
Myelination allows for the regulation of conduction velocity, affecting the precise timing of neuronal inputs important for the development and function of brain circuits. In turn, myelination may be altered by changes in experience, neuronal activity, and vesicular release, but the links between sensory experience, corresponding neuronal activity, and resulting alterations in myelination require further investigation. We thus studied the development of myelination in the Xenopus laevis tadpole, a classic model for studies of visual system development and function because it is translucent and visually responsive throughout the formation of its retinotectal system. We begin with a systematic characterization of the timecourse of early myelin ensheathment in the Xenopus retinotectal system using immunohistochemistry of myelin basic protein (MBP) along with third harmonic generation (THG) microscopy, a label-free structural imaging technique. Based on the mid-larval developmental progression of MBP expression in Xenopus, we identified an appropriate developmental window in which to assess the effects of early temporally patterned visual experience on myelin ensheathment. We used calcium imaging of axon terminals in vivo to characterize the responses of retinal ganglion cells over a range of stroboscopic stimulation frequencies. Strobe frequencies that reliably elicited robust versus dampened calcium responses were then presented to animals for $7 \mathrm{~d}$, and differences in the amount of early myelin ensheathment at the optic chiasm were subsequently quantified. This study provides evidence that it is not just the presence but also to the specific temporal properties of sensory stimuli that are important for myelin plasticity.
\end{abstract}

\section{KEYWORDS}

adaptive myelination, development, experience-dependent plasticity, neuron-glia interactions, third harmonic generation microscopy, visual system, Xenopus

This is an open access article under the terms of the Creative Commons Attribution License, which permits use, distribution and reproduction in any medium, provided 


\section{1 | INTRODUCTION}

The development and function of brain circuits relies crucially upon precise timing of neuronal inputs. By insulating axons to regulate the conduction velocity of inputs, myelination may optimize temporal control over information processing, with implications for synchrony in vertebrate circuits (Noori et al., 2020; Pajevic et al., 2014). An emerging hypothesis in the field is that dynamic control of conduction velocity by myelination may function as a potent form of metaplasticity, based on observations that myelination can be altered by changes in experience and neural activity (Chorghay et al., 2018; de Faria et al., 2018; Fields, 2015; Mount \& Monje, 2017; Purger et al., 2016; Xin $\&$ Chan, 2020). Effects of experience on myelination at the level of white matter changes studied with diffusion tensor imaging have been reported in animal and human brain imaging studies (Chorghay et al., 2018; Walhovd et al., 2014; Zatorre et al., 2012). On the other hand, investigations of cellular level changes have relied upon manipulations of axonal activity or vesicular release, including sensory deprivation, chronic pharmacological treatment, genetic manipulations, and electrical and optogenetic stimulation (Mount \& Monje, 2017; Xin \& Chan, 2020). Directly altering electrical activity and vesicular release in neurons modulates the number of myelinated segments (Demerens et al., 1996), lowers the expression and mobility of myelin-associated proteins such as myelin basic protein (MBP) (Wake et al., 2011), and affects the selection of axons for myelination as well as myelin sheath length (Hines et al., 2015; Mensch et al., 2015). Preventing myelination using genetic tools that target myelinating glia, such as oligodendrocytes in the rodent brain, has been shown to impair fear and spatial memory, demonstrating the importance of activity-dependent myelination for functional outcomes (Pan et al., 2020; Steadman et al., 2020; Wang et al., 2020).

The visual systems of rabbits and rodents have served as a model in which to probe how sensory experience directly alters myelination. Early eye opening of newborn animals accelerates myelination in the optic nerve (Ambronn, 1896; Tauber et al., 1980). Conversely, rearing mice in darkness leads to a reduced number of myelinated fibers compared with controls (Gyllensten \& Malmfors, 1963). Monocular deprivation results in a higher proportion of short myelin internodes and slower conduction velocity in the deprived optic nerve (Etxeberria et al., 2016). While the literature shows how artificial enhancement or deprivation may affect myelination, in the present study, we examined the effects of temporally patterned visual activity on myelination at early developmental stages by taking advantage of the Xenopus retinotectal system. This model system is amenable to imaging, shows precocious visual responsiveness, and has been extensively studied in the context of how patterned activity shapes synaptic plasticity, structural remodeling, and topographic circuit refinement (Demas et al., 2012; Kutsarova et al., 2016; Pratt et al., 2016). Temporally synchronized firing activity in retinal ganglion cells (RGCs) of animals reared under conditions of stroboscopic visual stimulation ("strobe") has been previously shown to disrupt topographic map development in the visual system, resulting in animals with expanded multiunit receptive fields, indicative of increased projection divergence of retinotectal and isthmotectal axonal inputs in Xenopus (Brickley et al., 1998) and of retinotectal axons in the goldfish (Schmidt \& Buzzard, 1993).

During visual system neurodevelopment of the Xenopus tadpole, RGC axons from the retina cross the midline at the chiasm and course towards the optic tectum at stage 34 (Gaze \& Peters, 1961). By stage 39 , which is the first stage at which a retinal response to light can be detected in the optic tectum, the axons have reached the tectum and begin arborization at coarsely topographically appropriate locations (Holt \& Harris, 1983). Subsequently, a precise retinotopic map in the optic tectum emerges, relying on visually mediated functional and structural plasticity mechanisms including spike-timing-dependent plasticity (Munz et al., 2014; Tsui et al., 2010; Zhang et al., 1998; Zhang et al., 2000). At these stages, presentation of visual stimuli that precede or follow the firing of tectal neurons by a matter of a just a few milliseconds can dynamically expand or shrink the visual receptive fields of these cells (Vislay-Meltzer et al., 2006). Furthermore, tadpoles transition from relying on their yolk sack for nutrition to active feeding at stage 47-48 (Nieuwkoop \& Faber, 1994), showing more complex sensorimotor behaviors in response to environmental cues. In light of the critical importance of input timing for circuit refinement and the role played by myelination in controlling conduction velocity, we examined myelination in the Xenopus visual system coinciding with this period of circuit development and plasticity.

In adult frogs, myelinated tracts have been extensively characterized (Llinás \& Precht, 1976). Just prior to adulthood, during metamorphosis, the frog optic nerve has been reported to undergo massive myelin remodeling (Cima \& Grant, 1982; Cullen \& Webster, 1979; Mills et al., 2015). However, there is limited literature on myelination in Xenopus prior to adulthood, so we first characterized the timecourse of early myelin ensheathment during mid-larval stages of development by immunostaining for MBP and cross-validating this with third harmonic generation (THG) microscopy. Since the links between sensory experience, corresponding neuronal activity, and myelination have yet to be fully elucidated, we next asked how sensory patterned activity alters myelination in the developing Xenopus retinotectal system. Specifically, we used calcium imaging to observe axonal activity induced by temporally patterned visual stimuli and examined MBP expression at the optic chiasm as a readout of changes in myelination.

\section{2 | METHODS}

\subsection{Animal husbandry}

All procedures were approved by the Animal Care Committee of the Montreal Neurological Institute at McGill University in accordance with Canadian Council on Animal Care guidelines. Tadpoles were raised in a $0.1 \times$ dilution of modified Barth's solution with HEPES (MBSH) $\left(88 \mathrm{mM} \mathrm{NaCl}, 1 \mathrm{mM} \mathrm{KCl}, 2.4 \mathrm{mM} \mathrm{NaHCO}_{3}, 0.82 \mathrm{mM} \mathrm{MgSO}_{4} \times 7 \mathrm{H}_{2} \mathrm{O}\right.$, $0.33 \mathrm{mM} \mathrm{Ca}\left(\mathrm{NO}_{3}\right)_{2} \times 4 \mathrm{H}_{2} \mathrm{O}, 0.41 \mathrm{mM} \mathrm{CaCl}{ }_{2}, 10 \mathrm{mM}$ HEPES, pH 7.4). For the MBP expression developmental timecourse, tadpoles were acquired from Boreal Science (RRID:XEP_Xla100). Upon receiving them, they were staged as per Nieuwkoop and Faber (1994) and immediately fixed for sectioning and immunostaining. 


\subsection{Western blot}

Samples were prepared from adult male Xenopus brain. After adult males were anesthetized in 0.2\% MS-222 (Sigma A5040) and decapitated, the brain was dissected and homogenized in RIPA extraction buffer (10 mM HEPES/ $\mathrm{NaOH}$ pH 7.4, $150 \mathrm{mM} \mathrm{NaCl}, 1 \%$ Triton X-100, 1\% SDS) with protease inhibitors (Calbiochem Protease Inhibitor Set V EDTA-free). Western blot analysis was performed with Bio-Rad wet transfer system and PVDF membrane (Millipore). Rat anti-MBP antibody (Abcam [clone 12] ab7349; RRID:AB_305869) was used at 1:5000 in 5\% skim milk, allowing the blot to incubate overnight at $4{ }^{\circ} \mathrm{C}$. Incubation with the secondary antibody, goat anti-rat HRP (Jackson ImmunoResearch 112-035-167; RRID:AB_2338139), was performed at 1:20,000 in 5\% skim milk for $1 \mathrm{~h}$ at room temperature. The blots were developed with Immobilon Western chemiluminescent HRP substrate (WBKLS0500).

\section{3 | Immunohistochemistry}

Animals were anesthetized in $0.02 \%$ MS-222, fixed by immersion in 4\% paraformaldehyde (Cedarlane (EMS) 15735-30-S) in PBS for $1 \mathrm{~h}$ at room temperature, transferred to ice-cold $100 \%$ methanol, and post-fixed overnight at $-20^{\circ} \mathrm{C}$. Samples were then washed for $1 \mathrm{~h}$ in a solution of $100 \mathrm{mM}$ Tris/ $\mathrm{HCl}, \mathrm{pH} 7.4$ with $100 \mathrm{mM} \mathrm{NaCl}$. Infiltration and cryoprotection of the samples were performed by incubation overnight at room temperature in a solution of $15 \%$ fish gelatin (Norland HP-03) with 15\% sucrose, and subsequently repeated in $25 \%$ fish gelatin with $15 \%$ sucrose. Samples were embedded and frozen in a solution of $20 \%$ fish gelatin with $15 \%$ sucrose for cryosectioning. Sections were acquired in the horizontal orientation at $20 \mu \mathrm{m}$ thickness on a cryostat and directly mounted onto Superfrost-plus slides (Fisher).

Slides were incubated with blocking solution (10\% bovine serum albumin and 5\% normal goat serum in PBS). Sections were immunostained with rat anti-MBP antibody (1:200; Abcam [clone 12] ab7349; RRID:AB_305869), mouse 3A10 (1:400; DSHB Hybridoma Product 3A10; RRID:AB_531874), and cell nuclei counterstained with DAPI (1:1000; Invitrogen D-1306; RRID:AB_2629482). The 3A10 monoclonal antibody is neuron-specific (Furley et al., 1990), preferentially labeling a subset of hindbrain spinal cord projecting neurons (Brand et al., 1996) and RGC axons (Manitt et al., 2009). The secondary antibodies used were goat anti-rat IgG Cy3 (1:200; Jackson ImmunoResearch 112-165-175; RRID:AB_2338252) and goat anti-mouse IgG Alexa-647 (1:200; Invitrogen A21236; RRID:AB_2535805). We used highly cross-adsorbed secondary antibodies in all instances to prevent cross-reactivity between mouse and rat primary antibodies. Images were acquired with $10 \times / 0.40 \mathrm{CS}, 40 \times / 1.30$ oil CS2, or $63 \times / 1.40$ oil CS2 objectives at a Leica SP8 confocal microscope. We investigated over 20 different antibodies and genetic constructs that had previously been used in mouse or zebrafish to label myelin, including reagents labeling PLP, CNP, O4, APC, Olig2, Caspr, NF186, Sox10, and NG2. Unfortunately, in our hands, only the MBP antibody (ab7349;
RRID:AB_305869) proved to be sufficiently specific and reliable to serve as a myelin marker in Xenopus larval tissue for our study.

\section{4 | THG microscopy}

THG imaging was performed on a custom-built laser scanning microscope with forward detection (as described in detail in Kazarine et al., 2019). The setup used a customized upright multiphoton microscope (FV1200 MPE; Olympus Canada Inc, ON, Canada) equipped with a motorized stage and a $25 \times$ water immersion objective (1.05 NA; 2 mm working distance; XLPL25XWMP(F), Olympus Canada Inc). Samples were excited by a Ti:Sapphire laser (Mira 900F; Coherent, Santa Clara, CA, USA) pumped by a $532 \mathrm{~nm}$ laser (Verdi V18; Coherent). The excitation laser provides $200 \mathrm{fs}$ pulses at a $76 \mathrm{MHz}$ repetition rate and feeds into an optical parametric oscillator (Mira OPO; Coherent), enabling $1150 \mathrm{~nm}$ pulses with a femtosecond pulse length necessary to serve the momentum conservation law (phase matching condition) for THG. A continuous power of $50 \mathrm{~mW}$ was measured at the plane of the sample. The emission light was split spectrally by a dichroic mirror (T425 Ipxr; Chroma Technology, Bellow Falls, VT, USA), separating third and second harmonic generation or respective two-photon emission, allowing for the simultaneous acquisition of two wavelengths with separate point detection on two photomultipliers for raster scan imaging. To detect THG signal, we collected the light through a 380-420 nm filter (ET400/40X; Chroma Technology), and to detect MBP immunostaining (secondary antibody $\mathrm{Cy} 3$ ), we used a 570-630 nm filter (ET600/60; Chroma Technology). Image stacks were acquired from $20 \mu \mathrm{m}$ thick horizontal sections immunostained for MBP (1:200 rat anti-MBP, 1:200 goat anti-rat Cy3). Images were denoised using CANDLE nonlocal means denoising software (Coupe et al., 2012) implemented in MATLAB (MathWorks).

\section{5 mRNA synthesis for blastomere injections}

To synthesize the messenger RNA (mRNA) for blastomere injections, GCaMP6s (Addgene plasmid 40753) and mCherry (plasmid gift of Dr Keith Murai) were each cloned into the pCS2+ vector. The GCaMP6s plasmid was cut with Notl/Klenow fill in/Bglll, the mCherry plasmid was cut with BamHI/EcoRV, and the pCS2+ vector was cut with BamH1 / SnaB1. The plasmids were linearized with Notl, and the capped mRNA of GCaMP6s and mCherry were transcribed with the SP6 mMessage mMachine Kit (Ambion; Thermo Fisher).

\subsection{Generation of tadpoles with bilateral mosaic expression of GCaMP6s}

Female albino Xenopus laevis frogs (RRID:XEP_Xla300) from our inhouse breeding colony were primed with $50 \mathrm{IU}$ pregnant mare serum gonadotropin (PMSG; Prospec Bio HOR-272), and 3 d later, were injected with 400 IU human chorionic gonadotropin (hCG; SigmaAldrich CG10; RRID:SCR_018232) to induce ovulation. The following 
day, eggs from primed females were collected for in vitro fertilization with sperm from male albino frogs and coinjection of GCaMP6s and mCherry mRNA into two-cell stage embryos as previously described (Kesner et al., 2020). Briefly, a mixture of purified GCaMP6s (500 pg) and mCherry (250 pg) mRNA in $2 \mathrm{nl}$ RNAase-free water was pressure injected into one blastomere of two-cell stage embryos using a calibrated glass micropipette attached to a PLI-100 picoinjector (Harvard Apparatus). Several days after injection, we screened for animals with unilateral mCherry and high levels of GCaMP fluorescence for use in calcium imaging experiments.

\subsection{Calcium imaging by two-photon microscopy}

Bilateral mosaic tadpoles expressing GCaMP6s at stage 48 were immobilized in $2 \mathrm{mM}$ pancuronium dibromide (Cedarlane/Tocris 0693) and embedded in $1 \%$ low melting point agarose in a petri dish filled with $0.1 \times \mathrm{MBSH}$. For visual stimulation, $10 \mathrm{~ms}$ full-field light flashes at different frequencies were generated with a red luxeon LED placed next to the petri dish and controlled by an Arduino UnoR3 board (RRID:SCR_017284). Animals were imaged with a 20x water-immersion objective (1.0 NA) mounted on a commercial highspeed resonance scanner-based multiphoton microscope (Thorlabs) with piezoelectric objective focusing (Physik Instruments). GCaMP6s fluorescence was excited using Spectra Physics InSight X2 femtosecond pulsed laser tuned to $910 \mathrm{~nm}$. Emission signal was detected with a GaAsP photomultiplier tube behind a 525/50 nm bandpass filter. Calcium signal was recorded from a single optical section ( $250 \mu \mathrm{m} \times 250 \mu \mathrm{m}$ imaging field), focusing on the neuropil region of one tectal hemisphere. Twenty seconds after initiating capture, the animal was shown a 1 min test stimulus (five flashes of 20 ms duration, presented $15 \mathrm{~s}$ apart), followed by 10 min of continuous LED flashes at the chosen frequency of stroboscopic stimulation (or no flashes for the spontaneous activity condition), immediately followed by another $1 \mathrm{~min}$ of the test stimulus. This was followed by a $5 \mathrm{~min}$ rest period between trials, then the stimulus sequence was repeated with the next strobe frequency being tested. The strobe frequencies tested were $0.0625,0.25,0.5,1,2$, and $4 \mathrm{~Hz}$.

\section{8 $\quad$ Strobe rearing}

For the strobe-rearing experiments, 2-3 d after priming females with 50 IU PMSG, 400 IU hCG was injected into females and 150 IU into males to induce mating. Fertilized eggs were collected the following day and once animals reached stage 48 , they were placed in a six-well plate in a box that blocked out external light, while exposed to a LED array composed of green luxeon LEDs controlled by a Master 8 Stimulator (A.M.P.I.). Animals were exposed to stroboscopic stimuli with $10 \mathrm{~ms}$ full-field light flashes presented at different frequencies or to constant stimulation at the same intensity ("ambient") for $7 \mathrm{~d}$. MBSH media and Sera-Micron $50 \mathrm{ml}$ growth food for fish and amphibians (Sera) was refreshed every 1-2 $d$.

\subsection{Quantification and statistical analysis}

\subsection{1 | Calcium imaging analysis}

Calcium recordings were registered using NoRMCorre (Pnevmatikakis \& Giovannucci, 2017), and analyzed using custom Matlab scripts. For each animal, the calcium signal was averaged over the neuropil region, the $\Delta F / F$ trace was calculated using the 20th percentile as baseline, then the signal corresponding to the strobe period was detrended with a fourth-degree polynomial before performing Fourier spectral analyses. The code for analysis of calcium responses to strobe stimuli can be found at https://github.com/RuthazerLab/Myelination Strobe-Ca2-Analysis.

\subsection{2 | MBP-masked 3A10 volume analysis}

Following immunohistochemical processing of tadpoles reared in strobe or ambient light for $7 \mathrm{~d}$, image stacks of each section containing the optic chiasm were exported to Imaris 9.2 (Bitplane) for 3D reconstruction of the chiasm and analysis in a blinded fashion. First, sequential stacks for each optic chiasm per animal were imported into the same file and manually aligned for 3D visualization. For each stack, the surface creation function in Imaris was used to automatically construct a surface from the MBP channel, setting the background subtraction with surface detail at $0.118 \mu \mathrm{m}$ and largest sphere diameter at $20 \mu \mathrm{m}$, thresholding automatically, with the final seed points approved by visual inspection. To study ensheathed axons at the chiasm, the 3A10 channel was masked with the MBP surface, and this masked channel was used to construct the MBP-masked 3A10 surface, using the same rendering parameters as the initial MBP surface. The volumes of the MBP-masked $3 \mathrm{~A} 10$ surface were summed to find the total volume of MBP-associated axons at the optic chiasm.

\subsection{3 | Statistical analysis}

Statistical analysis was performed in GraphPad Prism 8.0, and the details for each experiment can be found in the figure legend and figures. For the MBP-masked 3A10 volume, the ROUT test indicated one outlier (from $1 \mathrm{~Hz}$ strobe group) that was removed.

\section{3 | RESULTS}

\subsection{Investigation of early myelin ensheathment using MBP immunostaining}

To observe myelin ensheathment, we used an antibody against MBP, which showed expected band sizes of 19 and $22 \mathrm{kDa}$ on western blots of adult Xenopus brain lysate, in accordance with the reported molecular weights of MBP isoforms in Xenopus (Nanba et al., 2010) (Figure 1a). 
(a)

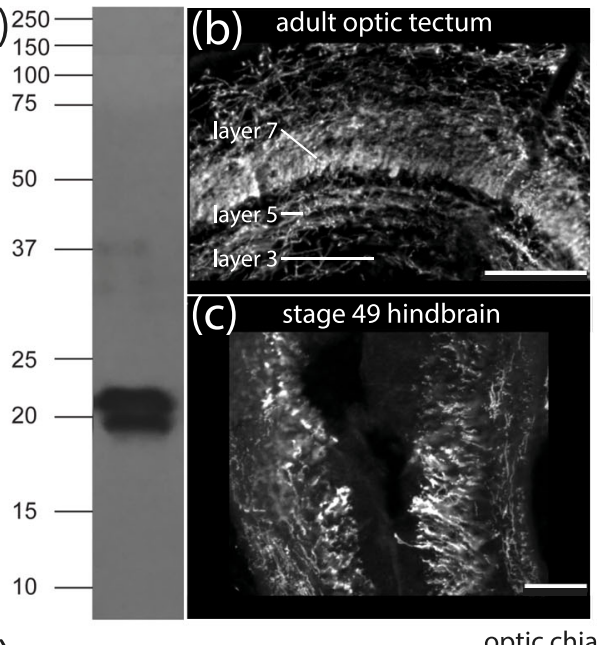

(e)

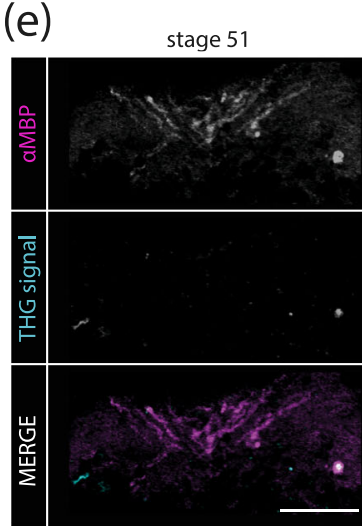

stage 52 optic chiasm
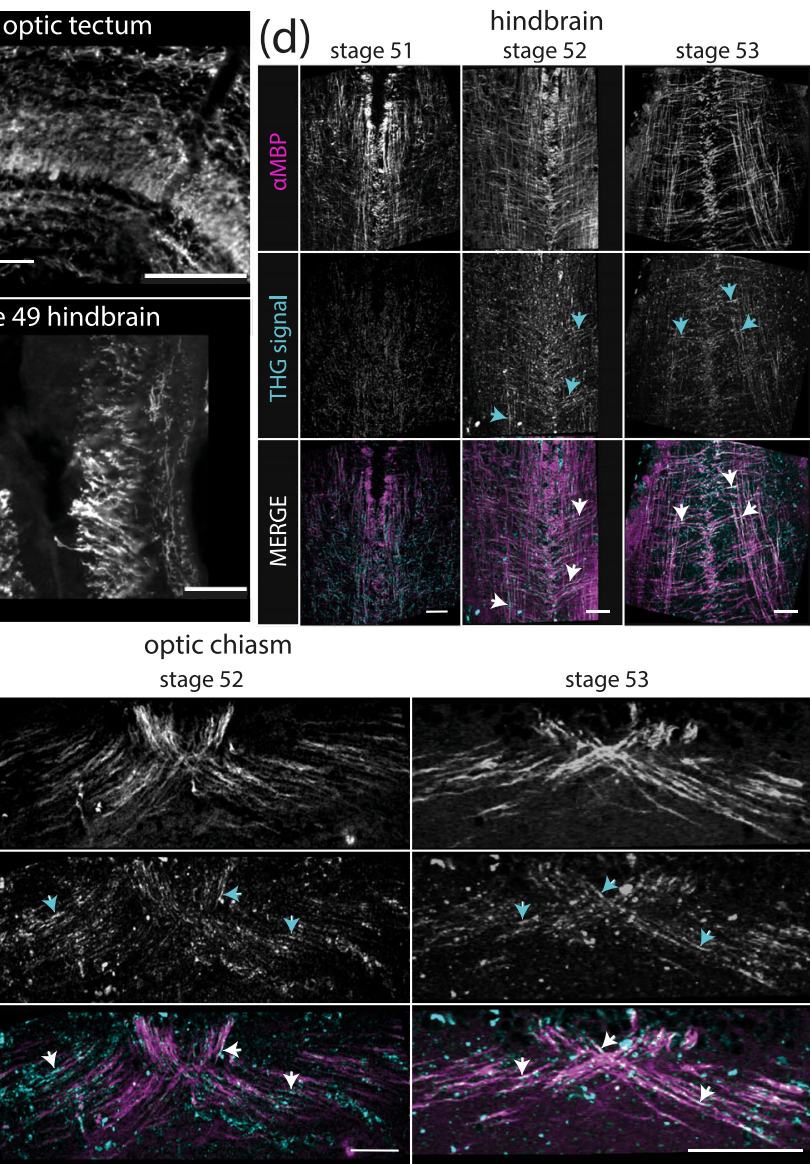

stage 53

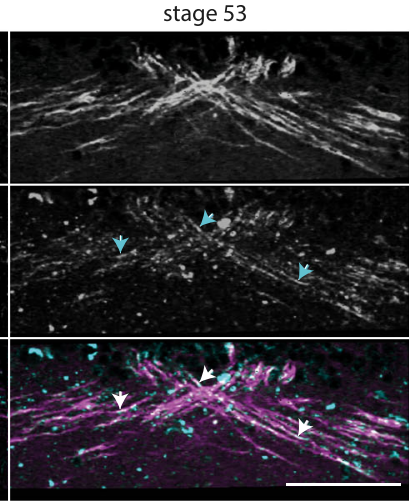

FIG URE 1 Investigation of early myelin ensheathment in the Xenopus laevis tadpole using MBP immunostaining and cross-validation with third harmonic generation (THG) microscopy. (a) Western blot using anti-MBP antibody on Xenopus adult brain lysate shows expected band sizes of approximately 19 and $22 \mathrm{kDa}$, corresponding to different MBP isoforms. (b and c) MBP immunofluorescence in (b) adult optic tectum, specifically in its plexiform layers 3, 5, and 7, and in (c) stage 49 hindbrain show expected morphology of myelinated fibers. Scale bar: $20 \mu \mathrm{m}$. (d and e) THG microscopy of the optic chiasm, showing two-photon MBP immunofluorescence aligned to THG signal in the same sections of tadpole stages 51-53. THG signal intensifies later in development than MBP expression, suggesting more compact myelin. Arrows: examples of fibers with both THG and MBP immunofluorescence signal. Colors: $\alpha$ MBP (magenta), THG (cyan). Scale bar: $40 \mu \mathrm{m}$. In this and subsequent figures, sections are horizontal and oriented with rostral toward the top of the panel

The pattern of MBP immunostaining reflected the laminar organization of the adult optic tectum with three plexiform layers (3, 5, and 7) interspersed between cellular layers (Lazar \& Szekely, 1967) (Figure 1b). In the hindbrain, the staining pattern was similar to immunostaining for the frog DM-20 isoform of myelin proteolipid protein reported in stage 49 tadpoles (Yoshida, 1997) (Figure 1c).

\section{2 | Cross-validation of MBP immunostaining with THG microscopy}

MBP immunostaining was cross-validated with THG microscopy (Figures $1 \mathrm{~d}$ and $1 \mathrm{e}$ ), an emerging label-free technique that has been used to image the presence of myelin in the peripheral and central nervous systems (Farrar et al., 2011; Lim et al., 2014). THG is a nonlinear optical process induced by the presence of an optical interface which breaks the symmetry across the focal volume to result in a measurable signal (Farrar et al., 2011). THG microscopy thus reveals submicrometer heterogeneities in refractive index, such as that observed in compact myelin where repeated transitions between lipid and cytoplasm occur, allowing it to be used as a structural imaging tool in unstained samples (Debarre, Pena, et al., 2006; Debarre, Supatto, et al., 2006). THG signal was observed in structures that exhibited MBP immunofluorescence within the hindbrain (Figure 1d) and optic chiasm (Figure 1e). Strong THG signal was observed in fewer fibers and at later development stages than MBP immunostaining, consistent with the progression of myelination from new ensheathment by MBP-positive processes to subsequent compaction which creates optical interfaces in the tissue for detection by THG microscopy (Farrar et al., 2011; Lim et al., 2014). For example, THG signal at the optic chiasm was not detected at stage 48 but was visible in older stage 52 animals, despite pronounced MBP expression throughout this developmental 


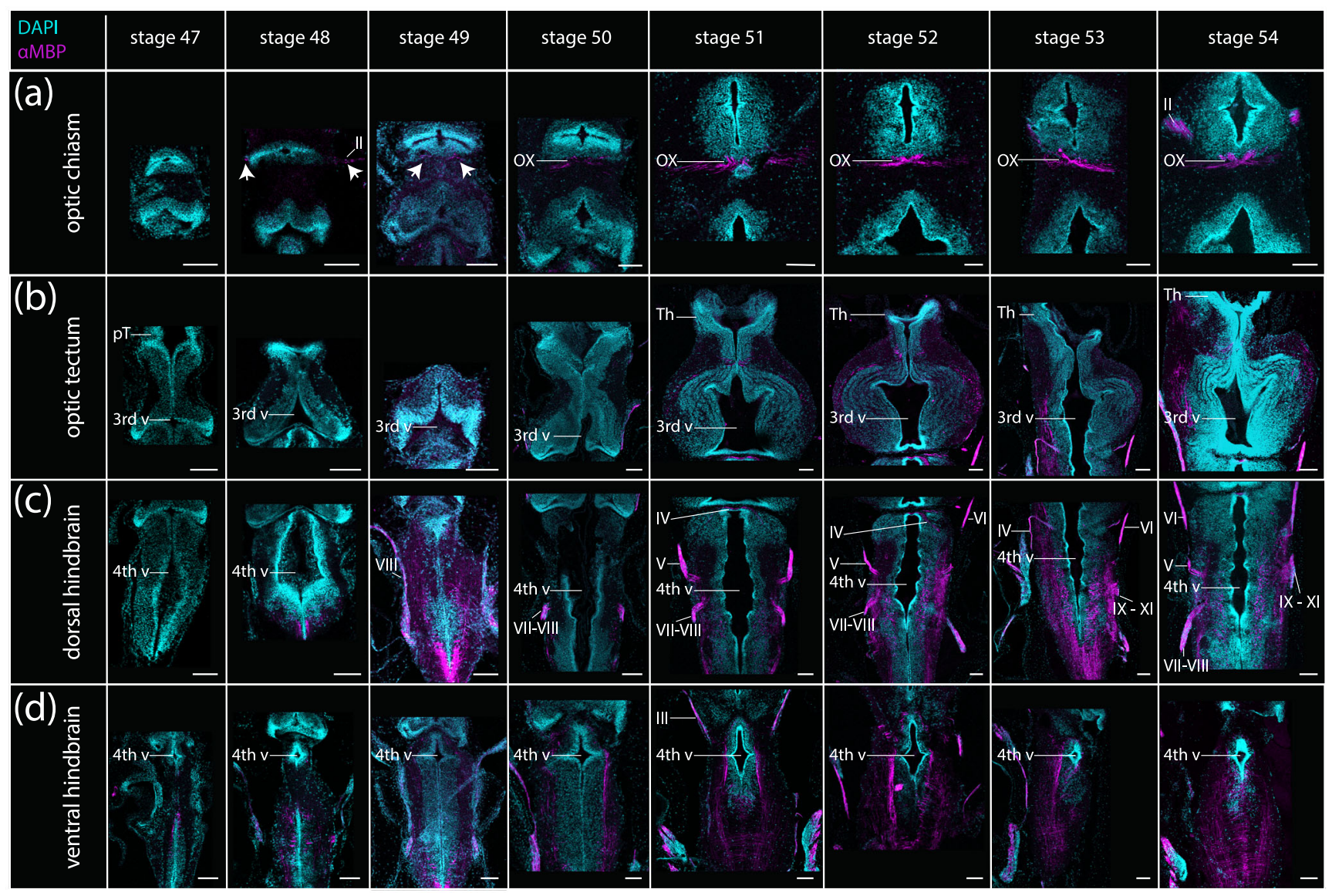

FIGURE 2 Caudal-to-rostral progression of MBP expression during neurodevelopment. MBP immunofluorescence in tadpoles from stages 47 to 54 observed in the (a) optic chiasm, (b) optic tectum, and the (c) dorsal and (d) ventral hindbrain. Arrows highlight MBP expression in the optic chiasm and nerve at stages with sparse immunostaining. Colors: $\alpha$ MBP (magenta), DAPI (cyan). Scale bar: $100 \mu$ m. Abbreviations: II, ON, optic nerve; III, oculomotor nerve; IV, trochlear nerve; V, trigeminal nerve; VI, abducens nerve; VII, facial nerve; VIII, vestibulocochlear nerve; X, vagus nerve; 3rd v, third ventricle; 4th v, fourth ventricle; OT, optic tectum; OX, optic chiasm; $\mathrm{pT}$, pretectum; Th, thalamus

time period. Because MBP expression was highly specific and preceded the onset of robust THG signal, we used MBP immunostaining for the rest of this study to investigate effects from the onset of myelination.

\section{3 | Caudal-to-rostral progression of MBP expression during neurodevelopment}

We examined MBP expression in mid-larval stage 47-54 tadpoles and observed immunostaining in the optic chiasm (Figure 2a) and tectum (Figure 2b), and the dorsal (Figure 2c) and ventral hindbrain (Figure 2d). MBP expression was observed to emerge in a caudal-to-rostral gradient in the developing Xenopus central nervous system, consistent with the developmental progression of myelination reported in other species, including rodents (Bjelke \& Seiger, 1989) and humans (Deoni et al., 2015; Flechsig, 1901). MBP expression was observed in the hindbrain and cranial nerves throughout mid-larval development, and in the optic nerve beginning at stage 48 , followed by the optic tectum beginning at stage 51 . While MBP immunostaining increased in these regions over subsequent stages, some brain regions like the olfactory bulb and the forebrain remained unmyelinated up to the oldest tadpole stages we examined.

\subsection{MBP expression in the developing Xenopus retinotectal system}

To investigate myelination in the larval retinotectal system, MBP was colabeled with 3A10 monoclonal antibody, which preferentially labels myelinating RGC axons (Manitt et al., 2009) as well as a subset of reticulospinal projections (Storey et al., 1992). We thus observed the timecourse of myelin ensheathment of axonal tracts in the visual system (Figure 3). We first saw MBP immunostaining at the optic chiasm at stage 48 (Figure 3a). An increase in MBP expression occurred throughout the developing brain at this time: differences were apparent between stage 48 (Figure 3b) and stage 51 (Figure 3c), not just in the optic nerve but also in the hindbrain (Figure 4) and optic tectum (Figure $3 d$ ). In the optic tectum, MBP expression begins by stage 51 and conforms to nongranular layers (Figure 3d) consistent with the 


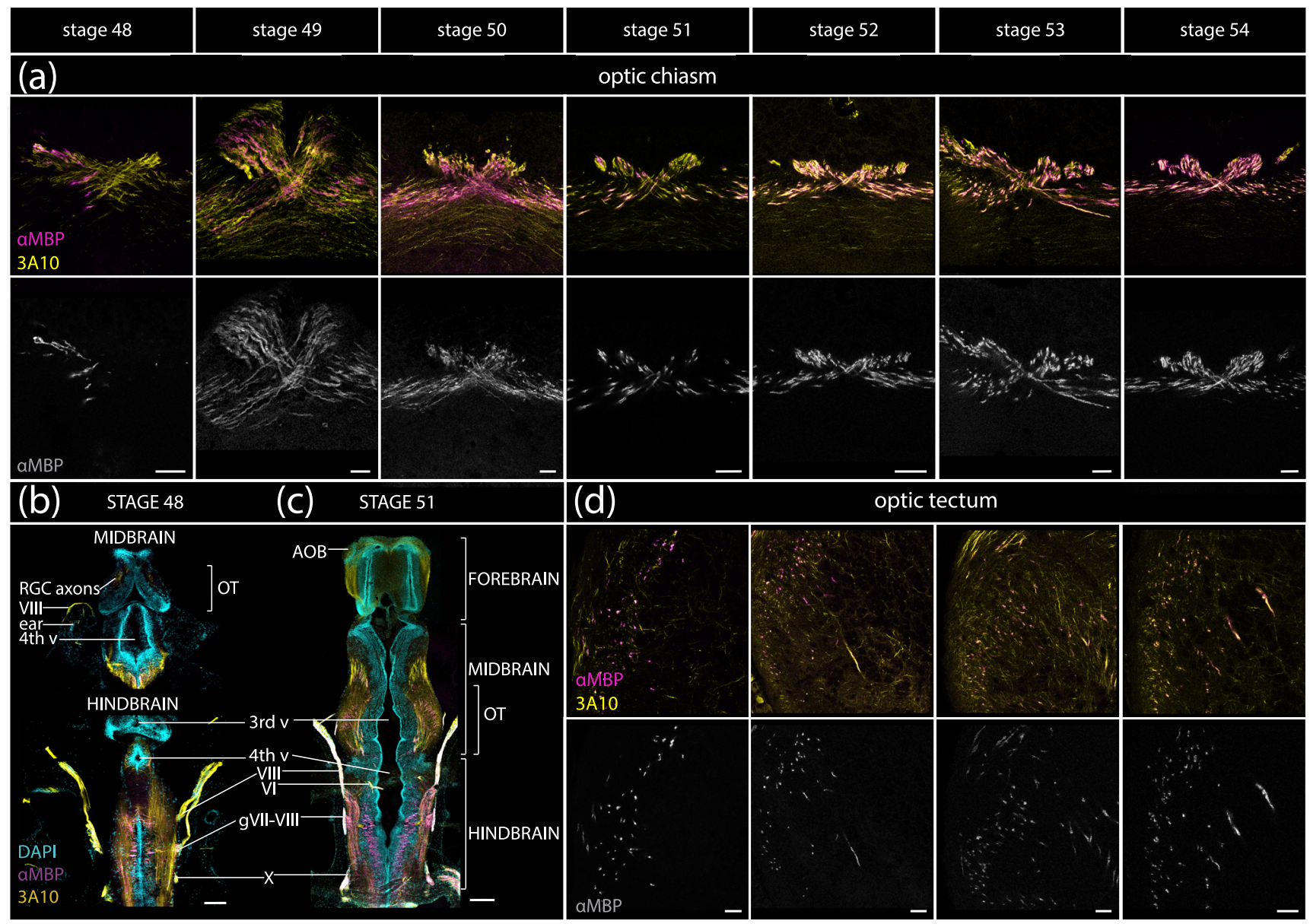

FIGURE 3 MBP expression in the developing Xenopus retinotectal system. (a) 3A10 labels RGC axons in the optic chiasm. MBP expression increases in the optic chiasm throughout tadpole development, beginning at stage 48 . Scale bar: $20 \mu \mathrm{m}$. (b and c) Myelination changes in the brain across the course of tadpole development between stages 48 and 51 . Scale bar: $100 \mu \mathrm{m}$. (d) In the optic tectum, MBP appears by stage 51 and is restricted to particular layers as the laminar organization of the tectum emerges. Scale bar: $20 \mu \mathrm{m}$. Colors: $\alpha$ MBP (magenta, gray), DAPI (cyan), 3A10 (yellow)

tectal laminar organization that emerges by adulthood (Lazar \& Szekely, 1969).

\subsection{Activity-dependent effects on MBP expression in the retinotectal system induced by stroboscopic stimulation}

Based on our characterization of the timecourse and location of early myelin ensheathment in the developing retinotectal system, we focused on the effects of visual experience on MBP expression at stage 48 in the optic chiasm. We used stroboscopic visual stimulation at different frequencies to physiologically induce temporally patterned activity in the retinotectal system (Figure 5a). To characterize the responses of the RGC axons to strobe, two-photon calcium imaging of RGC axon terminals in the optic tectum was performed. These axon terminals were labeled by injecting purified mRNA for GCaMP6s and $\mathrm{mCherry}$ into one blastomere of two-cell stage embryos, resulting in tadpoles with mosaic fluorescent protein expression restricted to one lateral half of the animal (Figure 5b) (Kesner et al., 2020). Since tadpole RGC axons cross over to the opposite tectum, RGC axons originating from the labeled side of the animal innervate the tectum on the unlabeled side. We recorded calcium activity in RGC axon terminals while presenting animals with strobe of varying frequencies from 0.0625 to $4 \mathrm{~Hz}$ (Figures 6b-6e) as well as spontaneous calcium activity measured in darkness (Figure 6a). We found that $0.0625 \mathrm{~Hz}$ ("slow strobe") reliably evoked robust calcium responses in RGC axons (Figures 5c and 5e), whereas $1 \mathrm{~Hz}$ ("fast strobe") generated only weak responses (Figures $5 d$ and $5 f$ ) in all of the animals tested. The Fourier power spectra for these calcium responses showed peaks corresponding to the frequencies for the slow (Figure 5g) and fast (Figure 5h) strobe conditions. Comparing the stimulus frequencies revealed significantly more power associated with the slow strobe than the fast strobe condition (Figure $5 \mathrm{i} ; n=3$, paired t-test, $p=.0034$ ), most likely due to the phenomenon of temporal frequency adaptation (Kim \& Rieke, 2001; Wark et al., 2007). We used these frequencies to assess the effects of different sensory stimuli on early myelination. 

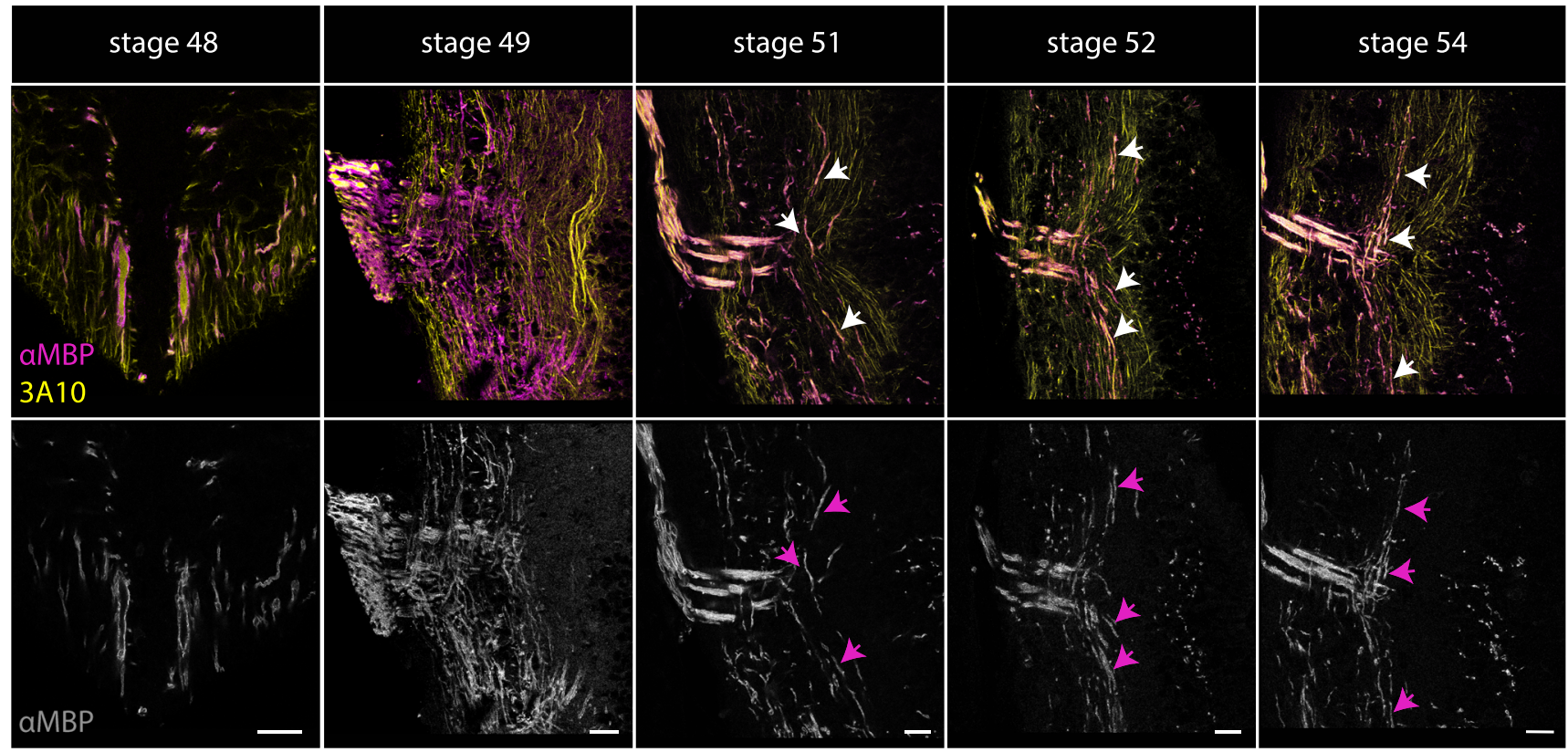

FIGURE 4 MBP expression in the developing Xenopus hindbrain. Arrows: MBP immunofluorescence in fibers of the hindbrain longitudinal tracts and cranial nerves. Colors: $\alpha$ MBP (magenta, gray), DAPI (cyan), 3A10 (yellow). Scale bar: $20 \mu \mathrm{m}$

We thus raised animals under slow strobe, fast strobe, or dim ambient light for $7 \mathrm{~d}$, after which animals were fixed and sectioned for immunostaining. We chose ambient light as the control condition rather than darkness as it has been previously shown that following dark rearing, RGCs in Xenopus can exhibit spontaneous local bursting that can drive patterns of correlated retinal activity, even in the absence of vision (Demas et al., 2012; Pratt et al., 2016). Tadpoles were fixed and immunostained, and high-resolution confocal stack images of the optic chiasm were acquired. From these photomontages, 3D digital reconstructions of $\mathrm{MBP}$ and $3 \mathrm{~A} 10$ profiles were used to quantify the total volume of MBP-associated RGC axons at the chiasm (Figures $5 j-5 m)$. The MBP-masked $3 A 10$ volume differed between the three conditions, with significantly greater axonal volume associated with MBP in animals reared under slow versus fast strobe (KruskalWallis test ${ }^{*} p=.0187$, Dunn's post-hoc test ${ }^{*} p=.0288$ ), while dim ambient light showed an intermediate level of myelin ensheathment (Figure 5j). Overall, our data show that slow strobe, which reliably evoked robust nonadapting calcium responses in RGC axons, increased the axonal volume associated with MBP, whereas fast strobe, which was associated with weak, adapting calcium responses, produced a reduction in ensheathed axonal volume at the chiasm.

\section{4 | DISCUSSION}

We performed a detailed characterization of the initial stages and progression of myelin ensheathment during Xenopus tadpole neurodevelopment by immunostaining for MBP and with THG microscopy. In the visual system, myelination begins at the optic chiasm around the time of intensive timing-dependent plasticity in the retinotectal system. At this crucial developmental window (stage 48), we examined links between sensory experience, axonal activity, and myelination. Animals exposed to stroboscopic visual stimuli of varying frequencies showed robust versus dampened calcium responses in their RGC axons and corresponding differences in myelination at the optic chiasm. Specifically, RGC axons exposed to slow strobe showed robust calcium responses and increased volume associated with MBP, as compared with fast strobe, which led to dampened calcium responses and relatively decreased ensheathed axon volume. Therefore, we show that myelination can be modulated by specific patterns of sensory stimulation and the strength of neuronal activation itself (which varies over different stimulus frequencies), together suggesting that differential neuronal activity induced by sensory temporally patterned visual stimulation regulates developmental myelination.

Despite considerable research on how patterned activity controls synaptic plasticity and circuit refinement, elucidating the role of glia in activity-dependent circuit development is a relatively recent endeavor in the field. To study these neuron-glia interactions during circuit development, we took advantage of the Xenopus retinotectal system, which has classically served as a model for understanding the role of sensory visual experience and patterned activity in structural and functional topographic circuit refinement (Demas et al., 2012; Kutsarova et al., 2016; Pratt et al., 2016). Immunostaining for MBP revealed a caudal-to-rostral developmental progression of MBP expression in the tadpole brain. Across species, including in humans, myelination progresses from the brainstem to sensorimotor to association areas (Deoni et al., 2015; Flechsig, 1901). 
(a)

(c)

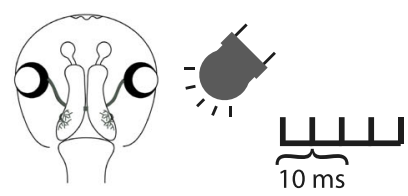

slow strobe $(0.0625 \mathrm{~Hz}$ stimulation)

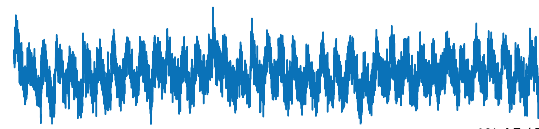

(e)

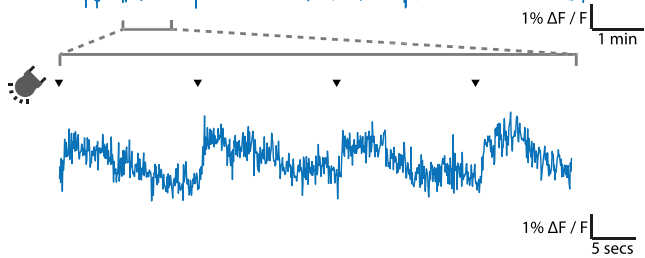

(g)

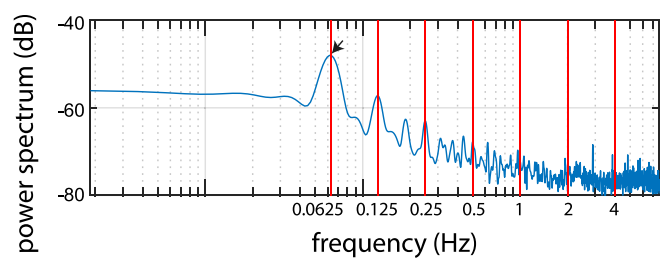

(b)

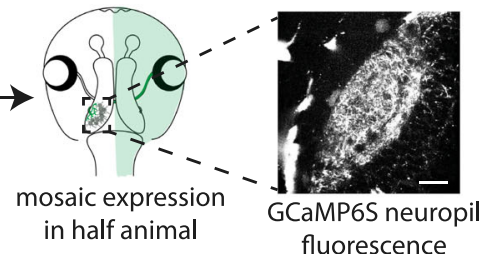

fast strobe (1 $\mathrm{Hz}$ stimulation)

(d)

(f)

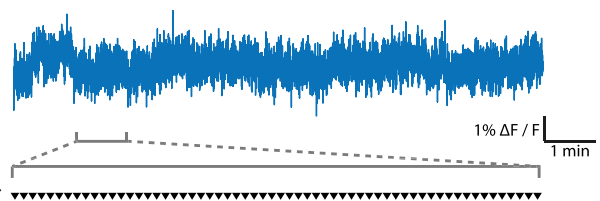

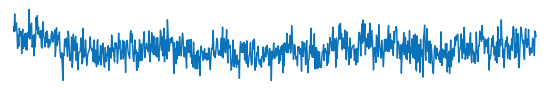

(h)
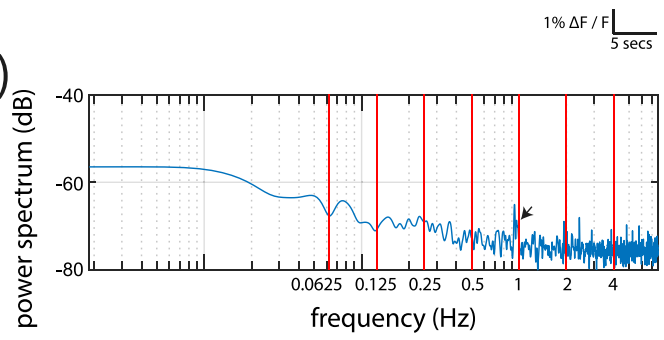

(i)

(j)

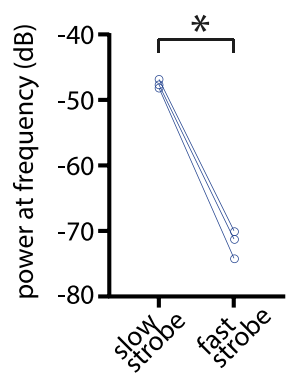

(k)
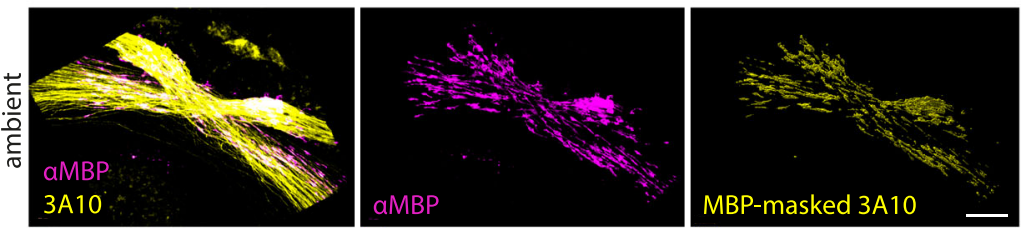

(I)
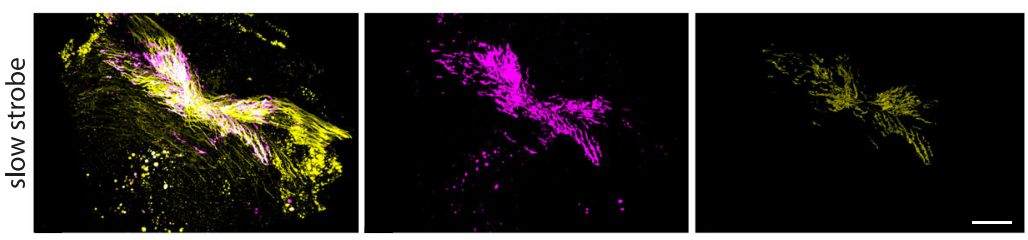

(m)
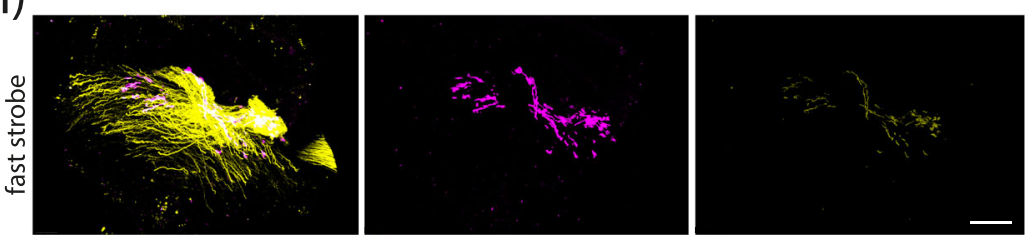

FIGURE 5 Activity-dependent effects on MBP expression in the retinotectal system with stroboscopic visual stimulation. (a) Xenopus tadpoles were exposed to $10 \mathrm{~ms}$ stroboscopic flashes at a range of frequencies. (b) For two-photon calcium imaging of RGC axon terminals in the optic tectum, tadpoles with bilateral mosaic GCaMP6s expression restricted to half the animal were generated by microinjection of GCaMP6s mRNA into one blastomere at the two-cell stage of development. Scale bar: $40 \mu \mathrm{m}$. (c and d) Representative tectal neuropil axonal calcium responses to the $(c)$ slow strobe $(0.0625 \mathrm{~Hz})$ and $(\mathrm{d})$ fast strobe $(1 \mathrm{~Hz})$ stimuli over $10 \mathrm{~min}$ and zoomed in to a (e and f) 1 min period. Arrowhead: LED flashes during stroboscopic visual stimulation. ( $g$ and $h$ ) Fourier power spectra of calcium responses in (c and d). Arrow: peak in power corresponding to strobe frequency. (i) Power at the stimulus frequency was significantly greater for slow rather than fast strobe in each animal $\left(n=3\right.$, paired t-test, $\left.{ }^{*} p=.0034\right)$. ( $\mathrm{j}-\mathrm{m}$ ) Animals were raised under ambient light, slow or fast strobe for $7 \mathrm{~d}$, and their optic chiasm digitally reconstructed in 3D. (j) Quantification of the MBP-masked 3A10 volume showed differences between the three conditions. ( $n=9$ for ambient, 7 for slow strobe, 6 for fast strobe. Line: median. Kruskal-Wallis test: ${ }^{*} p=.0187$, Dunn's test for multiple comparisons: ${ }^{*} p=.0288$ for slow vs. fast strobe.) (k-m) Representative snapshots of the reconstructions for the (k) ambient, (l) slow, and ( $m$ ) fast strobe conditions. Colors: $\alpha M B P$ (magenta), DAPI (cyan), 3A10 (yellow). Scale bar: $20 \mu \mathrm{m}$ 
(a)

raw $\mathrm{Ca}^{2+} \operatorname{trace}(1 \% \Delta \mathrm{F} / \mathrm{F})$

(b)

(c)
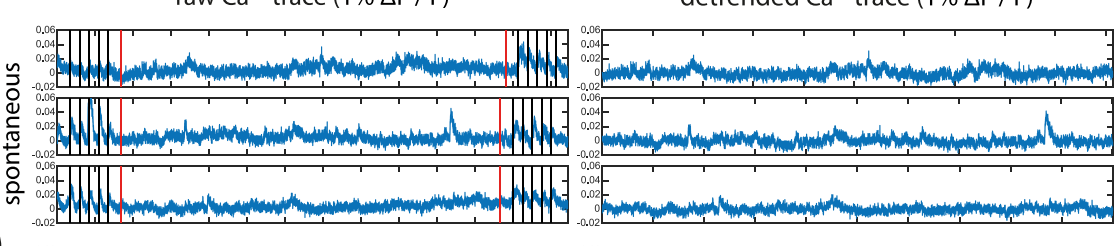

detrended $\mathrm{Ca}^{2+}$ trace $(1 \% \Delta \mathrm{F} / \mathrm{F})$
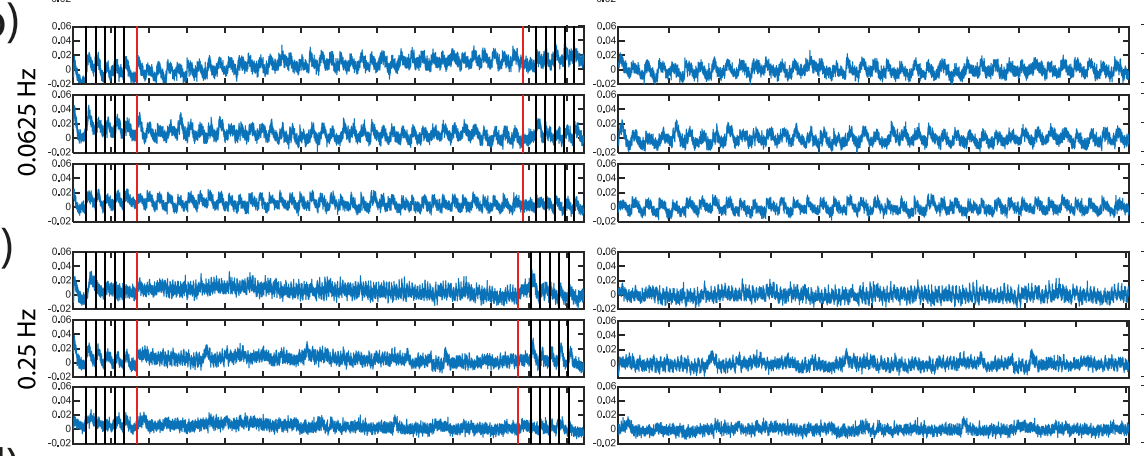

(d)
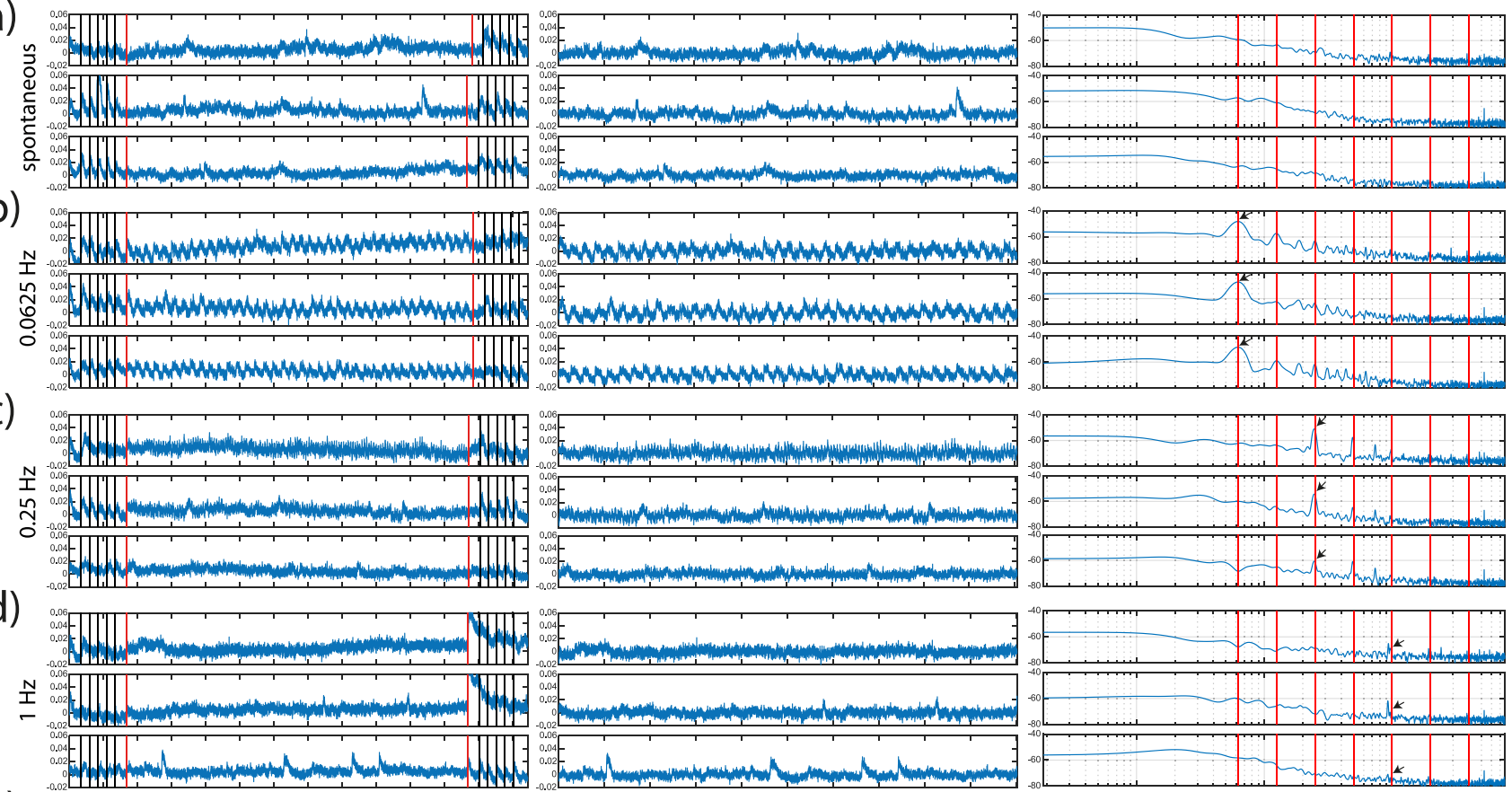

(e)
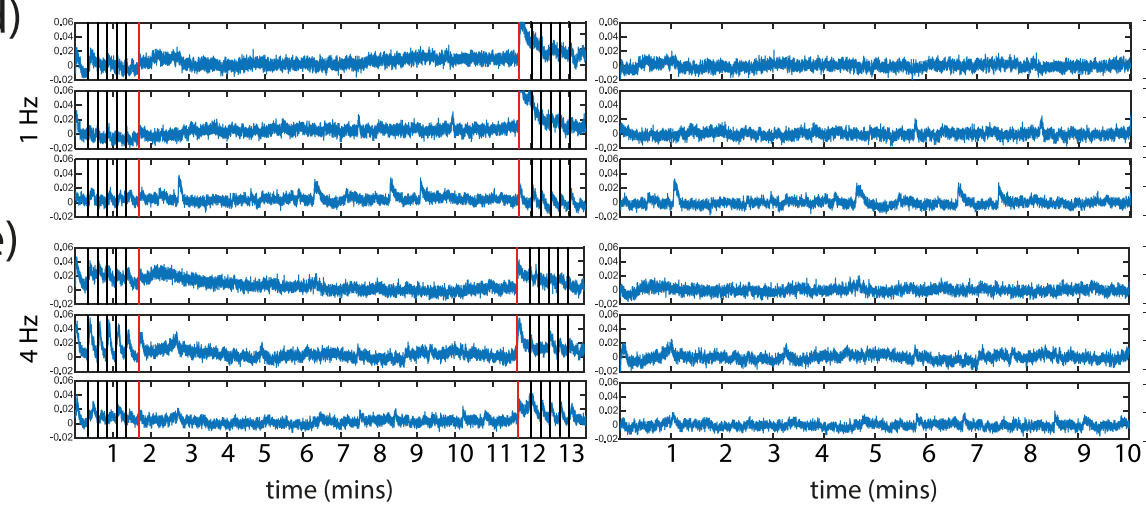

Fourier power spectrum (dB)
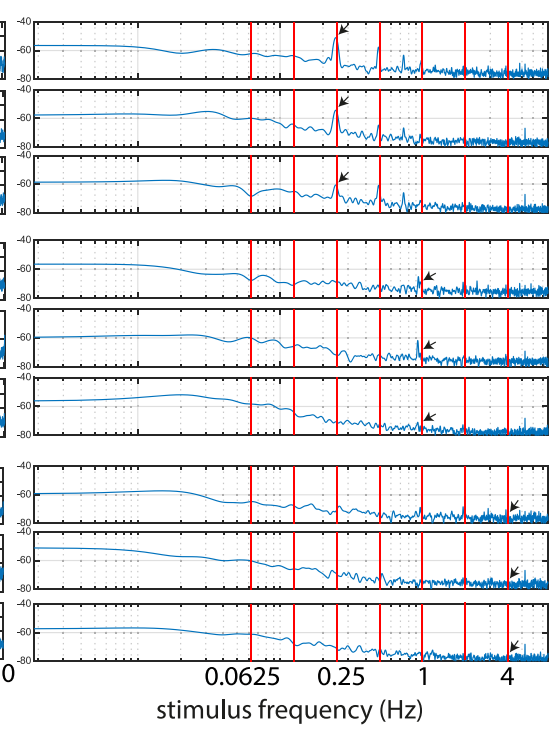

FIGURE 6 RGC axon calcium responses in the optic tectum in response to stroboscopic stimulation of different frequencies. Different strobe frequencies were tested in order to identify stimuli that led to robust versus dampened activity in RGC axons, including (a) no strobe condition from which we recorded spontaneous calcium responses, (b) $0.0625 \mathrm{~Hz}$ strobe which showed robust activity, (c) $0.25 \mathrm{~Hz}$ strobe, (d) $1 \mathrm{~Hz}$ strobe, and (e) $4 \mathrm{~Hz}$ strobe, which appeared to show increasing dampening of neuronal activity. Left column: raw $\Delta F / F$ traces of the calcium response to strobe show mean neuropil responses during the strobe stimulation period (red bars: start and end of this period) as well as immediately before and after in response to test stimuli (5 LED flashes of $20 \mathrm{~ms}$ duration each, presented $15 \mathrm{~s}$ apart, indicated by black vertical bars). The large response to the test stimuli following strobe stimulation suggests adaptation of RGCs during high-frequency strobe stimulation, which recovered rapidly. Middle column: calcium traces detrended with a fourth-degree polynomial. Right column: corresponding Fourier power spectra $(n=3$ animals; in respective rows)

One caveat of using MBP expression to study myelination is that although MBP is present specifically in myelinating oligodendrocytes (Ackerman \& Monk, 2016), neither MBP nor any markers are known to be specific for detecting compact myelin, which can thus far only be confirmed by electron microscopy. We therefore cross-validated MBP immunostaining with THG microscopy, a technique that reveals sub-micrometer heterogeneities produced at optical interfaces such as those present at transitions between lipid and cytoplasm, allowing it to be used as a structural imaging tool to visualize myelination in unstained samples (Debarre, Pena, et al., 2006; Debarre, Supatto, et al., 2006). Only a handful of studies have used this method to study myelination in any species (Farrar et al., 2011; Lim et al., 2014). In contrast to MBP immunostaining, THG signal is detected in fewer fibers and at later developmental stages, suggesting that it may be detecting more compacted myelin.
MBP expression was observed in the hindbrain and the cranial nerves throughout the mid-larval development stages we examined. The earliest stage in which oligodendrocytes have been reported in Xenopus is stage 42 in the hindbrain (Yoshida, 1997), and the hindbrain continues to myelinate throughout development in order to form the many longitudinal and transverse myelinated fibers observed in adulthood (Nieuwenhuys, 1976). We next observed MBP expression in the optic nerve at stage 48, particularly prominent at the optic chiasm, consistent with electron microscopy studies reporting optic nerve myelination first occurring at stage 46 (Wilson, 1971), 48/49 (Cima \& Grant, 1982), or 49 (Gaze \& Peters, 1961). These slight differences in the stage at which myelin first appears in the optic nerve likely arose from how different researchers stage Xenopus tadpoles, and from variability between animals (Grant \& Rubin, 1980). In the adult X. laevis, the proportion of myelinated fibers in the optic nerve towards the periphery 
has been reported as $16 \%$ (Wilson, 1971) or 15\%, and increased substantially to $96 \%$ at the chiasm (Cima \& Grant, 1982). The optic tectum is the most studied optic center in the amphibian brain, showing a laminar organization classified into nine layers in the adult, with alternating cellular and plexiform layers, with layers 3,5 , and 7 consisting of fiber tracts while layer 9 consists of cellular and plexiform sublayers (Lazar \& Szekely, 1969). Consistent with forming this laminar structure, MBP expression in the optic tectum first appeared at stage 50 and was restricted to particular layers as the laminae become more distinctly visible in subsequent stages. Finally, in the mid-larval stages we examined, up to stage 54, we failed to see any MBP expression anterior to the optic chiasm. Even in adulthood, the forebrain fiber systems including the olfactory tracts, lateral and medial forebrain bundles, precommissural fornix, and stria medullaris are thin and largely unmyelinated (Scalia, 1976).

The differences among and within regions in MBP expression throughout development and persisting into adulthood observed in Xenopus and other species reflect the ability of vertebrate circuits to use myelination to confer heterogeneity in axonal conduction velocities (Pajevic et al., 2014). For example, the mouse thalamocortical projections of the somatosensory system show intra- and inter-fiber differences in their myelination states and conduction velocities (Salami et al., 2003). Similarly, differences in myelination are important for timing in the circuits of the auditory hindbrain, enabling accurate sound localization in the avian (Seidl et al., 2010) and mammalian (Ford et al., 2015) brain. This heterogeneity is not entirely intrinsic, since beyond genetic programs that initiate myelination of medium and large-diameter axons, neuronal activity and experience can finetune the myelination state of axons (Bechler et al., 2018). Key evidence for this phenomenon of adaptive myelination or myelin plasticity came from a number of studies that have shown that altering neuronal activity can impact the differentiation and proliferation of myelinating glia and of myelination itself (Demerens et al., 1996; Hughes et al., 2018; Lundgaard et al., 2013; Makinodan et al., 2012; Mitew et al., 2018; Stevens et al., 2002; Wake et al., 2011; Wang et al., 2020; Yang et al., 2020), which in turn affects learning and memory (Bacmeister et al., 2020; Gibson et al., 2014; Kato et al., 2020; McKenzie et al., 2014; Steadman et al., 2020; Xiao et al., 2016). In the present study, we aimed to establish the links between sensory experience, corresponding neuronal activity, and alterations in myelination.

Patterned visual stimulation in vivo, designed to systematically alter the degree of correlation in the firing of retinotectal inputs, has previously been shown to direct structural and functional refinement of the retinotectal circuit. In Xenopus, strobe rearing synchronizes RGC firing and enlarges multiunit receptive fields, while preventing the activitydependent alignment of binocular inputs from the nucleus isthmi (Brickley et al., 1998). In goldfish exposed to $1 \mathrm{~Hz}$ strobe stimuli, axonal arbors become enlarged and diffuse, with atypically large response fields compared with controls (Schmidt \& Buzzard, 1993; Schmidt \& Eisele, 1985). Presentation of strobe stimuli leading to synchronous activation of RGC arbors decreased branch additions, whereas asynchronous activation increased branch formation (Munz et al., 2014; Rahman et al., 2020). By imaging the RGC calcium responses evoked by strobe stimuli at varying frequencies, we were able to show that temporally patterned sensory visual stimulation elicits different types of neuronal activity, that is, slow strobe $(0.0625 \mathrm{~Hz})$ evoked robust calcium activity in RGCs whereas relatively fast strobe $(1 \mathrm{~Hz})$ dampened neuronal activity likely due to temporal frequency adaptation (Kim \& Rieke, 2001; Wark et al., 2007).

In terms of myelin plasticity, the effects of the frequency of axonal firing on myelination have previously been tested in co-cultures of Schwann cells and dorsal root ganglion neurons, wherein $0.1 \mathrm{~Hz}$ but not $1 \mathrm{~Hz}$ electrical stimulation reduced MBP expression (Stevens et al., 1998). Similarly, different frequencies of electrical stimulation have been shown to differentially regulate myelination in the corpus callosum (Nagy et al., 2017). Our finding that the frequency of sensory stimulation differentially affected MBP expression at the optic chiasm is consistent with this observation. Although our study identified an increase in the volume of ensheathed RGC axons was associated with lower rather than higher stimulus frequencies, these experiments demonstrate reduced myelination under conditions where lower levels of axonal firing might be anticipated.

How glia may detect and respond to neuronal activity has yet to be elucidated. It has been proposed that glia sense molecules, including the neurotransmitter glutamate, released from active axons, either at sites of contact or through spontaneous release (Kukley et al., 2007; Wake et al., 2015; Ziskin et al., 2007), then respond with electrical activity (Berret et al., 2017; Karadottir et al., 2008) and calcium influx in glial processes and myelin sheaths (Baraban et al., 2018; Krasnow et al., 2018), leading to signaling cascades underlying myelination. When vesicular release is prevented in single axons by tetanus toxin expression, they are less likely to be selected for myelination, and those that are myelinated have reduced sheath lengths compared with controls (Hines et al., 2015; Mensch et al., 2015). This inhibition of sheath growth is thought be competitive, since it is relieved by suppressing the activity of neighboring axons with administration of the voltage-gated $\mathrm{Na}+$ channel blocker, tetrodotoxin (Hines et al., 2015). Conversely, stimulation of spinal cord axons in the zebrafish affects calcium transients in contacting oligodendrocytes, which then predict myelin sheath dynamics (Baraban et al., 2018; Krasnow et al., 2018), reminiscent of correlations between calcium and structural dynamics of neurons for activity-dependent plasticity. Given these findings, and that even engineered nanofibers can be wrapped in myelin as long as they have an appropriate diameter (Lee et al., 2012), a twophase model of intrinsic and adaptive myelination has been proposed. According to this model, initial indiscriminate myelination is followed by the activity-dependent phase, where myelinating glia detect levels of neuronal activity and accordingly select and refine myelin ensheathment of these axons (Bechler et al., 2018). In our study, some level of myelination was seen in all three conditions of visual experience, suggesting that initial myelination occurs but is influenced by patterned sensory and axonal activation. Furthermore, specific patterns of neuronal activity are known to induce distinct programs of gene expression (Tyssowski et al., 2018), thus different stimulation frequencies could conceivably lead to changes in expression of genes associated with myelination, including MBP. Our findings lend support to the 
possibility of shared mechanisms for the control of timing-dependent plasticity and myelination during circuit development.

Interestingly, it has also been proposed that differential myelination may reflect activity-dependent changes in axonal morphology such as the elaboration or stability of the axonal arbor. In the medial prefrontal cortex, parvalbumin interneurons show progressively decreasing myelination with increasing branch order (Stedehouder et al., 2018). When these neurons are stimulated using Designer Receptors Exclusively Activated by Designer Drug, they show an increase in total myelination of more distal and higher order axonal branches, which the researchers suggest alludes to the activity-dependent increase in myelination of parvalbumin interneurons being predominantly mediated by the elaboration of axon morphology. In a separate study of parvalbumin interneurons in the primary visual cortex of monocularly deprived mice, myelin remodeling was observed on stable axonal branches rather than on axonal branches that exhibited experiencedependent changes in length (Yang et al., 2020). Since we studied myelination along the axonal tract at the optic chiasm through which axons extend with minimal branch elaboration, changes in MBP expression here are unlikely to be influenced by axonal branch morphogenesis, though it is possible that the resultant changes in conduction velocity could influence branch dynamics at the terminal arbors where spiketiming-dependent synaptic plasticity occurs (Munz et al., 2014; Tsui et al., 2010; Vislay-Meltzer et al., 2006; Zhang et al., 1998; Zhang et al., 2000).

Taken together, we studied myelination and myelin plasticity in the context of a developing circuit by first characterizing its normal developmental progression, and then altering it during a developmentally relevant time window through sensory-evoked patterned activity. By regulating the conduction velocity of action potentials, myelin alterations may contribute to network synchrony and oscillations (Pajevic et al., 2014), as has been shown for motor learning (Kato et al., 2020) and by computational modeling wherein changes in conduction velocity through adaptive myelination allow for more robust synchronization of the network than could be accounted for by synaptic gain alone (Noori et al., 2020). Even small changes in conduction can synchronize or desynchronize the timing of spikes to affect network oscillations with functional consequences, for example, for memory consolidation during sleep (Buzsaki, 1996; Diekelmann et al., 2010; Peyrache et al., 2009). How myelinating glia may sense and respond to neuronal activity to coordinate differential myelination for the dynamic control of conduction velocity, and therefore, the temporal synchrony of circuits, is an important future direction for this work and for the larger field of myelin plasticity. Last, disorders such as addiction, autism, bipolar disorder, depression, and schizophrenia (Bartzokis et al., 2010; Fields, 2008) have been linked to myelin abnormalities, along with a dysregulation of temporal synchrony, highlighting the importance of experience-dependent myelination in circuit development and refinement.

\section{ACKNOWLEDGMENTS}

Thank you to Dr Nicholas Marsh-Armstrong for suggesting the antiMBP and 3A10 antibodies for use in Xenopus, the Neuro Microscopy
Core Facility for access to the Leica SP8 microscope, and the Advanced Bioimaging Facility at McGill University for access to analysis on Imaris, particularly Dr Nelly Vuillemin for her expertise with this software. We also thank Drs Alyson Fournier, Tim Kennedy, and Barbara Morquette for their mentorship and guidance regarding myelination.

This work was funded by a chaire de recherche from the Fonds de recherche du Québec - Santé (FRQS Grant 31036) to E. S. R., the Montreal Neurological Institute - University of Cambridge Collaboration Grant to R. T. K. and E. S. R., the European Union's Horizon 2020 Research and Innovation Programme (Grant Agreement No 771411) through the European Research Council (ERC) to R. T. K., the Wellcome Trust [203151/Z/16/Z] and the UKRI Medical Research Council [MC_PC_17230] to R. T. K., the Natural Sciences and Engineering Research Council of Canada (NSERC) Discovery Grant to P. W. W., the NSERC Canada Graduate Scholarship - Master's and McGill University Integrated Program in Neuroscience Studentships to Z. C. and V. J. L., and the Douglas Avrith Award to Z. C.

\section{CONFLICT OF INTEREST}

The authors declare no competing interests.

\section{DATA AVAILABILITY STATEMENT}

The data that support the findings of this study are available from the corresponding author upon reasonable request.

\section{PEER REVIEW}

The peer review history for this article is available at https://publons. com/publon/10.1002/cne.25253.

\section{ORCID}

Edward S. Ruthazer (D) https://orcid.org/0000-0003-0452-3151

\section{REFERENCES}

Ackerman, S. D., \& Monk, K. R. (2016). The scales and tales of myelination: Using zebrafish and mouse to study myelinating glia. Brain Research, 1641(Pt A), 79-91. https://doi.org/10.1016/j.brainres.2015.10.011

Ambronn, H., \& Held, H. (1896). I. Über Entwicklung und Bedeutung des Nervenmarks. Beiträge zur Kenntnis des Nervenmarks. Leipzig: Arch. Anat. Physiol., 202-213.

Bacmeister, C. M., Barr, H. J., Mcclain, C. R., Thornton, M. A., Nettles, D., Welle, C. G., \& Hughes, E. G. (2020). Motor learning promotes remyelination via new and surviving oligodendrocytes. Nature Neuroscience, 23(7), 819-831. https://doi.org/10.1038/s41593-020-0637-3

Baraban, M., Koudelka, S., \& Lyons, D. A. (2018). Ca (2+) activity signatures of myelin sheath formation and growth in vivo. Nature Neuroscience, 21(1), 19-23. https://doi.org/10.1038/s41593-017-0040-x

Bartzokis, G., Lu, P.o H., Tingus, K., Mendez, M. F., Richard, A., Peters, D. G., Oluwadara, B., Barrall, K. A., Finn, J. P., Villablanca, P., Thompson, P. M., \& Mintz, J. (2010). Lifespan trajectory of myelin integrity and maximum motor speed. Neurobiology of Aging, 31(9), 1554-1562. https://doi.org/ 10.1016/j.neurobiolaging.2008.08.015

Bechler, M. E., Swire, M., \& Ffrench-Constant, C. (2018). Intrinsic and adaptive myelination-A sequential mechanism for smart wiring in the brain. Developmental Neurobiology, 78(2), 68-79. https://doi.org/10. 1002/dneu.22518

Berret, E., Barron, T., Xu, J., Debner, E., Kim, E. J., \& Kim, J. H. (2017). Oligodendroglial excitability mediated by glutamatergic inputs and Nav1.2 
activation. Nature communications, 8(1), 557. https://doi.org/10.1038/ s41467-017-00688-0

Bjelke, B., \& Seiger, Å. (1989). Morphological distribution of MBP-like immunoreactivity in the brain during development. International Journal of Developmental Neuroscience, 7(2), 145-164. https://doi.org/10.1016/ 0736-5748(89)90065-8

Brand, M., Heisenberg, C. P., Jiang, Y. J., Beuchle, D., Lun, K., Furutani-Seiki, M., Granato, M., Haffter, P., Hammerschmidt, M., Kane, D. A., Kelsh, R. N., Mullins, M. C., Odenthal, J., Van Eeden, F. J., \& Nusslein-Volhard, C. (1996). Mutations in zebrafish genes affecting the formation of the boundary between midbrain and hindbrain. Development (Cambridge, England), 123, 179-190.

Brickley, S. G., Dawes, E. A., Keating, M. J., \& Grant, S. (1998). Synchronizing retinal activity in both eyes disrupts binocular map development in the optic tectum. Journal of Neuroscience, 18(4), 1491-1504.

Buzsáki, G. (1996). The hippocampo-neocortical dialogue. Cerebral Cortex, 6(2), 81-92. https://doi.org/10.1093/cercor/6.2.81

Chorghay, Z., Káradóttir, R. T., \& Ruthazer, E. S. (2018). White matter plasticity keeps the brain in tune: Axons conduct while glia wrap. Front Cell Neurosci, 12, 428. https://doi.org/10.3389/fncel.2018.00428

Cima, C., \& Grant, P. (1982). Development of the optic nerve in Xenopus laevis. II. Gliogenesis, myelination and metamorphic remodelling. Journal of Embryology and Experimental Morphology, 72, 251-267.

Code for analysis available at: https://github.com/RuthazerLab/ Myelination_Strobe-Ca2-Analysis

Coupé, P., Munz, M., Manjón, J. V., Ruthazer, E. S., \& Louis Collins, D. (2012). A CANDLE for a deeper in vivo insight. Medical Image Analysis, 16(4), 849864. https://doi.org/10.1016/j.media.2012.01.002

Cullen, M. J., \& Def Webster, H. (1979). Remodelling of optic nerve myelin sheaths and axons during metamorphosis in Xenopus laevis. Journal of Comparative Neurology, 184(2), 353-362. https://doi.org/10.1002/cne. 901840209

de Faria, O., Pama, E. A. C., Evans, K., Luzhynskaya, A., \& Káradóttir, R. T. (2018). Neuroglial interactions underpinning myelin plasticity. Developmental Neurobiology, 78(2), 93-107. https://doi.org/10.1002/dneu. 22539

Débarre, D., Pena, A.-M., Supatto, W., Boulesteix, T., Strupler, M., Sauviat, M. P., Martin, J.-L., Schanne-Klein, M.-C., \& Beaurepaire, E. (2006). [Secondand third-harmonic generation microscopies for the structural imaging of intact tissues.]. Medecine Sciences, 22(10), 845-852. https://doi.org/10. 1051/medsci/20062210845

Débarre, D., Supatto, W., Pena, A.-M., Fabre, A., Tordjmann, T., Combettes, L., Schanne-Klein, M.-C., \& Beaurepaire, E. (2006). Imaging lipid bodies in cells and tissues using third-harmonic generation microscopy. Nature Methods, 3(1), 47-53. https://doi.org/10.1038/nmeth813

Demas, J. A., Payne, H., \& Cline, H. T. (2012). Vision drives correlated activity without patterned spontaneous activity in developing Xenopus retina. Developmental Neurobiology, 72(4), 537-546. https://doi.org/10.1002/ dneu. 20880

Demerens, C., Stankoff, B., Logak, M., Anglade, P., Allinquant, B., Couraud, F., Zalc, B., \& Lubetzki, C. (1996). Induction of myelination in the central nervous system by electrical activity. Proceedings of the National Academy of Sciences of the United States of America, 93(18), 9887-9892. https://doi. org/10.1073/pnas.93.18.9887

Deoni, S. C. L., Dean, D. C., Remer, J., Dirks, H., \& O'muircheartaigh, J. (2015). Cortical maturation and myelination in healthy toddlers and young children. Neuroimage, 115, 147-161. https://doi.org/10.1016/j.neuroimage. 2015.04.058

Diekelmann, S., Born, J., \& Wagner, U. (2010). Sleep enhances false memories depending on general memory performance. Behavioural Brain Research, 208(2), 425-429. https://doi.org/10.1016/j.bbr.2009.12.021

Etxeberria, A., Hokanson, K. C., Dao, D. Q., Mayoral, S. R., Mei, F., Redmond, S. A., Ullian, E. M., \& Chan, J. R. (2016). Dynamic modulation of myelination in response to visual stimuli alters optic nerve conduction veloc- ity. Journal of Neuroscience, 36(26), 6937-6948. https://doi.org/10.1523/ JNEUROSCI.0908-16.2016

Farrar, M. J., Wise, F. W., Fetcho, J. R., \& Schaffer, C. B. (2011). In vivo imaging of myelin in the vertebrate central nervous system using third harmonic generation microscopy. Biophysical Journal, 100(5), 1362-1371. https:// doi.org/10.1016/j.bpj.2011.01.031

Fields, R. D. (2008). White matter in learning, cognition and psychiatric disorders. Trends in Neuroscience (Tins), 31(7), 361-370. https://doi.org/10. 1016/j.tins.2008.04.001

Fields, R. D. (2015). A new mechanism of nervous system plasticity: Activitydependent myelination. Nature Reviews Neuroscience, 16(12), 756-767. https://doi.org/10.1038/nrn4023

Flechsig Of Leipsic, P. (1901). Developmental (myelogenetic) localisation of the cerebral cortex in the human subject. Lancet, 158(4077), 1027-1030. https://doi.org/10.1016/S0140-6736(01)01429-5

Ford, M. C., Alexandrova, O., Cossell, L., Stange-Marten, A., Sinclair, J., KoppScheinpflug, C., Pecka, M., Attwell, D., \& Grothe, B. (2015). Tuning of Ranvier node and internode properties in myelinated axons to adjust action potential timing. Nature Communications, 6, 8073. https://doi.org/ 10.1038/ncomms9073

Furley, A. J., Morton, S. B., Manalo, D., Karagogeos, D., Dodd, J., \& Jessell, T. M. (1990). The axonal glycoprotein TAG-1 is an immunoglobulin superfamily member with neurite outgrowth-promoting activity. Cell, 61(1), 157-170. https://doi.org/10.1016/0092-8674(90)90223-2

Gaze, R. M., \& Peters, A. (1961). The development, structure and composition of the optic nerve of Xenopus laevis (Daudin). Quarterly Journal of Experimental Physiology and Cognate Medical Sciences, 46, 299-309. https: //doi.org/10.1113/expphysiol.1961.sp001548

Gibson, E. M., Purger, D., Mount, C. W., Goldstein, A. K., Lin, G. L., Wood, L. S., Inema, I., Miller, S. E., Bieri, G., Zuchero, J. B., Barres, B. A., Woo, P. J., Vogel, H., \& Monje, M. (2014). Neuronal activity promotes oligodendrogenesis and adaptive myelination in the mammalian brain. Science, 344(6183), 1252304. https://doi.org/10.1126/science.1252304

Grant, P., \& Rubin, E. (1980). Ontogeny of the retina and optic nerve in Xenopus laevis. II. Ontogeny of the optic fiber pattern in the retina. Journal of Comparative Neurology, 189(4), 671-698. https://doi.org/10.1002/cne. 901890405

Gyllensten, L., \& Malmfors, T. (1963). Myelinization of the optic nerve and its dependence on visual function-a quantitative investigation in mice. Journal of Embryology and Experimental Morphology, 11, 255-266.

Hines, J. H., Ravanelli, A. M., Schwindt, R., Scott, E. K., \& Appel, B. (2015) Neuronal activity biases axon selection for myelination in vivo. Nature Neuroscience, 18(5), 683-689. https://doi.org/10.1038/nn.3992

Holt, C. E., \& Harris, W. A. (1983). Order in the initial retinotectal map in Xenopus: A new technique for labelling growing nerve fibres. Nature, 301(5896), 150-152. https://doi.org/10.1038/301150a0

Hughes, E. G., Orthmann-Murphy, J. L., Langseth, A. J., \& Bergles, D. E. (2018). Myelin remodeling through experience-dependent oligodendrogenesis in the adult somatosensory cortex. Nature Neuroscience, 21(5), 696-706. https://doi.org/10.1038/s41593-018-0121-5

Káradóttir, R., Hamilton, N. B., Bakiri, Y., \& Attwell, D. (2008). Spiking and nonspiking classes of oligodendrocyte precursor glia in CNS white matter. Nature Neuroscience, 11(4), 450-456. https://doi.org/10.1038/ nn2060

Kato, D., Wake, H., Lee, P. R., Tachibana, Y., Ono, R., Sugio, S., Tsuji, Y., Tanaka, Y. H., Tanaka, Y. R., Masamizu, Y., Hira, R., Moorhouse, A. J., Tamamaki, N., Ikenaka, K., Matsukawa, N., Fields, R. D., Nabekura, J., \& Matsuzaki, M. (2020). Motor learning requires myelination to reduce asynchrony and spontaneity in neural activity. Glia, 68(1), 193-210. https://doi.org/10. 1002/glia.23713

Kazarine, A., Gopal, A. A., \& Wiseman, P. W. (2019). Nonlinear microscopy of common histological stains reveals third harmonic generation harmonophores. Analyst, 144(10), 3239-3249. https://doi.org/10.1039/ c9an00267g 
Kesner, P., Schohl, A., Warren, E. C., Ma, F., \& Ruthazer, E. S. (2020). Postsynaptic and presynaptic NMDARs have distinct roles in visual circuit development. Cell Reports, 32(4), 107955. https://doi.org/10.1016/ j.celrep.2020.107955

Kim, K. J., \& Rieke, F. (2001). Temporal contrast adaptation in the input and output signals of salamander retinal ganglion cells. Journal of Neuroscience, 21(1), 287-299.

Krasnow, A. M., Ford, M. C., Valdivia, L. E., Wilson, S. W., \& Attwell, D. (2018). Regulation of developing myelin sheath elongation by oligodendrocyte calcium transients in vivo. Nature Neuroscience, 21(1), 24-28. https://doi. org/10.1038/s41593-017-0031-y

Kukley, M., Capetillo-Zarate, E., \& Dietrich, D. (2007). Vesicular glutamate release from axons in white matter. Nature Neuroscience, 10(3), 311-320. https://doi.org/10.1038/nn1850

Kutsarova, E., Munz, M., \& Ruthazer, E. S. (2016). Rules for shaping neural connections in the developing brain. Frontiers in Neural Circuits, 10, 111. https://doi.org/10.3389/fncir.2016.00111

Lat'Zat'R, G. Y., \& Szet'Kely, G. Y. (1969). Distribution of optic terminals in the different optic centres of the frog. Brain Research, 16(1), 1-14. https: //doi.org/10.1016/0006-8993(69)90081-x

Lazar, G., \& Szekely, G. (1967). Golgi studies on the optic center of the frog. Journal Fur Hirnforschung, 9(4), 329-344.

Lee, S., Leach, M. K., Redmond, S. A., Chong, S. Y. C., Mellon, S. H., Tuck, S. J., Feng, Z.-Q.i, Corey, J. M., \& Chan, J. R. (2012). A culture system to study oligodendrocyte myelination processes using engineered nanofibers. Nature Methods, 9(9), 917-922. https://doi.org/10.1038/nmeth.2105

Lim, H., Sharoukhov, D., Kassim, I., Zhang, Y., Salzer, J. L., \& MelendezVasquez, C. V. (2014). Label-free imaging of Schwann cell myelination by third harmonic generation microscopy. Proceedings of the National Academy of Sciences of the United States of America, 111(50), 1802518030. https://doi.org/10.1073/pnas.1417820111

Llinás, R., \& Precht, W. E. (1976). Frog Neurobiology. Heidelberg, Germany: Springer-Verlag Berlin.

Lundgaard, I., Luzhynskaya, A., Stockley, J. H., Wang, Z., Evans, K. A., Swire, M., Volbracht, K., Gautier, H. O. B., Franklin, R. J. M., Ffrench-Constant, C., Attwell, D., \& Káradóttir, R. T. (2013). Neuregulin and BDNF induce a switch to NMDA receptor-dependent myelination by oligodendrocytes. PLoS Biology, 11(12), e1001743. https://doi.org/10.1371/journal. pbio.1001743

Makinodan, M., Rosen, K. M., Ito, S., \& Corfas, G. (2012). A critical period for social experience-dependent oligodendrocyte maturation and myelination. Science, 337(6100), 1357-1360. https://doi.org/10.1126/science. 1220845

Manitt, C., Nikolakopoulou, A. M., Almario, D. R., Nguyen, S. A., \& CohenCory, S. (2009). Netrin participates in the development of retinotectal synaptic connectivity by modulating axon arborization and synapse formation in the developing brain. Journal of Neuroscience, 29(36), 1106511077. https://doi.org/10.1523/JNEUROSCI.0947-09.2009

Mckenzie, I. A., Ohayon, D., Li, H., Paes De Faria, J., Emery, B., Tohyama, K., \& Richardson, W. D. (2014). Motor skill learning requires active central myelination. Science, 346(6207), 318-322. https://doi.org/10.1126/ science. 1254960

Mensch, S., Baraban, M., Almeida, R., Czopka, T., Ausborn, J., El Manira, A., \& Lyons, D. A. (2015). Synaptic vesicle release regulates myelin sheath number of individual oligodendrocytes in vivo. Nature Neuroscience, 18(5), 628-630. https://doi.org/10.1038/nn.3991

Mills, E. A., Davis, C.-H.a O., Bushong, E. A., Boassa, D., Kim, K.-Y., Ellisman, M. H., \& Marsh-Armstrong, N. (2015). Astrocytes phagocytose focal dystrophies from shortening myelin segments in the optic nerve of Xenopus laevis at metamorphosis. Proceedings of the National Academy of Sciences of the United States of America, 112(33), 10509-10514. https://doi.org/ 10.1073/pnas.1506486112

Mitew, S., Gobius, I., Fenlon, L. R., Mcdougall, S. J., Hawkes, D., Xing, Y. L., Bujalka, H., Gundlach, A. L., Richards, L. J., Kilpatrick, T. J., Merson, T.
D., \& Emery, B. (2018). Pharmacogenetic stimulation of neuronal activity increases myelination in an axon-specific manner. Nature Communications, 9(1), 306. https://doi.org/10.1038/s41467-017-02719-2

Mount, C. W., \& Monje, M. (2017). Wrapped to adapt: Experiencedependent myelination. Neuron, 95(4), 743-756. https://doi.org/10. 1016/j.neuron.2017.07.009

Munz, M., Gobert, D., Schohl, A., Poquerusse, J., Podgorski, K., Spratt, P., \& Ruthazer, E. S. (2014). Rapid Hebbian axonal remodeling mediated by visual stimulation. Science, 344(6186), 904-909. https://doi.org/10. 1126/science. 1251593

Nagy, B., Hovhannisyan, A., Barzan, R., Chen, T.-J., \& Kukley, M. (2017). Different patterns of neuronal activity trigger distinct responses of oligodendrocyte precursor cells in the corpus callosum. PLoS Biology, 15(8), e2001993. https://doi.org/10.1371/journal.pbio.2001993

Nanba, R., Fujita, N., \& Nagata, S. (2010). Structure and expression of myelin basic protein gene products in Xenopus laevis. Gene, 459(1-2), 32-38. https://doi.org/10.1016/j.gene.2010.03.010

Nieuwenhuys, R. O., \& P. (1976). Structure of the Brain Stem. In (R. P. Llinás, W. Ed.), Frog Neurobiology (pp. 811-847). Heidelberg, German: SpringerVerlag Berlin.

Nieuwkoop, P. D., \& Faber, J. (1994). Normal table of Xenopus laevis (Daudin).

Noori, R., Park, D., Griffiths, J. D., Bells, S., Frankland, P. W., Mabbott, D., \& Lefebvre, J. (2020). Activity-dependent myelination: A glial mechanism of oscillatory self-organization in large-scale brain networks. Proceedings of the National Academy of Sciences of the United States of America, 117(24), 13227-13237. https://doi.org/10.1073/pnas.1916646117

Pajevic, S., Basser, P. J., \& Fields, R. D. (2014). Role of myelin plasticity in oscillations and synchrony of neuronal activity. Neuroscience, 276, 135-147. https://doi.org/10.1016/j.neuroscience.2013.11.007

Pan, S., Mayoral, S. R., Choi, H. S., Chan, J. R., \& Kheirbek, M. A. (2020). Preservation of a remote fear memory requires new myelin formation. Nature Neuroscience, 23(4), 487-499. https://doi.org/10.1038/ s41593-019-0582-1

Peyrache, A., Khamassi, M., Benchenane, K., Wiener, S. I., \& Battaglia, F. P. (2009). Replay of rule-learning related neural patterns in the prefrontal cortex during sleep. Nature Neuroscience, 12(7), 919-926. https://doi.org/ 10.1038/nn.2337

Pnevmatikakis, E. A., \& Giovannucci, A. (2017). NoRMCorre: An online algorithm for piecewise rigid motion correction of calcium imaging data. Journal of Neuroscience Methods, 291, 83-94. https://doi.org/10.1016/j. jneumeth.2017.07.031

Pratt, K. G., Hiramoto, M., \& Cline, H. T. (2016). An evolutionarily conserved mechanism for activity-dependent visual circuit development. Frontiers in Neural Circuits, 10, 79. https://doi.org/10.3389/fncir.2016.00079

Purger, D., Gibson, E. M., \& Monje, M. (2016). Myelin plasticity in the central nervous system. Neuropharmacology, 110(Pt B), 563-573. https://doi. org/10.1016/j.neuropharm.2015.08.001

Rahman, T. N., Munz, M., Kutsarova, E., Bilash, O. M., \& Ruthazer, E. S. (2020). Stentian structural plasticity in the developing visual system. Proceedings of the National Academy of Sciences of the United States of America, 117(20), 10636-10638. https://doi.org/10.1073/pnas.2001107117

Salami, M., Itami, C., Tsumoto, T., \& Kimura, F. (2003). Change of conduction velocity by regional myelination yields constant latency irrespective of distance between thalamus and cortex. Proceedings of the National Academy of Sciences of the United States of America, 100(10), 6174-6179. https://doi.org/10.1073/pnas.0937380100

Scalia, F. (1976). Structure of the Olfactory and Accessory Olfactory System. In (R. P. Llinás, W. Ed.), Frog Neurobiology (pp. 213-232). Heidelberg, German: Springer-Verlag Berlin.

Schmidt, J. T., \& Buzzard, M. (1993). Activity-driven sharpening of the retinotectal projection in goldfish: Development under stroboscopic illumination prevents sharpening. Journal of Neurobiology, 24(3), 384-399. https://doi.org/10.1002/neu.480240310 
Schmidt, J. T., \& Eisele, L. E. (1985). Stroboscopic illumination and dark rearing block the sharpening of the regenerated retinotectal map in goldfish. Neuroscience, 14(2), 535-546. https://doi.org/10.1016/0306-4522(85) 90308-2

Seidl, A. H., Rubel, E. W., \& Harris, D. M. (2010). Mechanisms for adjusting interaural time differences to achieve binaural coincidence detection. Journal of Neuroscience, 30(1), 70-80. https://doi.org/10.1523/ JNEUROSCI.3464-09.2010

Steadman, P. E., Xia, F., Ahmed, M., Mocle, A. J., Penning, A. R. A., Geraghty, A. C., Steenland, H. W., Monje, M., Josselyn, S. A., \& Frankland, P. W. (2020). Disruption of oligodendrogenesis impairs memory consolidation in adult mice. Neuron, 105(1), 150-164.e6. https://doi.org/10.1016/ j.neuron.2019.10.013

Stedehouder, J., Brizee, D., Shpak, G., \& Kushner, S. A. (2018). Activitydependent myelination of parvalbumin interneurons mediated by axonal morphological plasticity. Journal of Neuroscience, 38(15), 3631-3642. https://doi.org/10.1523/JNEUROSCI.0074-18.2018

Stevens, B., Porta, S., Haak, L. L., Gallo, V., \& Fields, R. D. (2002). Adenosine: A neuron-glial transmitter promoting myelination in the CNS in response to action potentials. Neuron, 36(5), 855-868. https://doi.org/10.1016/ s0896-6273(02)01067-x

Stevens, B., Tanner, S., \& Fields, R. D. (1998). Control of myelination by specific patterns of neural impulses. Journal of Neuroscience, 18(22), 93039311.

Storey, K. G., Crossley, J. M., De Robertis, E. M., Norris, W. E., \& Stern, C. D. (1992). Neural induction and regionalisation in the chick embryo. Development (Cambridge, England), 114(3), 729-741.

Tauber, H., Waehneldt, T. V., \& Neuhoff, V. (1980). Myelination in rabbit optic nerves is accelerated by artificial eye opening. Neuroscience Letters, 16(3), 235-238. https://doi.org/10.1016/0304-3940(80)90003-8

Tsui, J. (2010). A developmental sensitive period for spike timing-dependent plasticity in the retinotectal projection. Front Synaptic Neurosci, 2, 13. https://doi.org/10.3389/fnsyn.2010.00013

Tyssowski, K. M., Destefino, N. R., Cho, J.-H., Dunn, C. J., Poston, R. G., Carty, C. E., Jones, R. D., Chang, S. M., Romeo, P., Wurzelmann, M. K., Ward, J. M., Andermann, M. L., Saha, R. N., Dudek, S. M., \& Gray, J. M. (2018). Different neuronal activity patterns induce different gene expression programs. Neuron, 98(3), 530-546.e11. https://doi.org/10.1016/j.neuron.2018.04. 001

Vislay-Meltzer, R. L., Kampff, A. R., \& Engert, F. (2006). Spatiotemporal specificity of neuronal activity directs the modification of receptive fields in the developing retinotectal system. Neuron, 50(1), 101-114. https://doi.org/10.1016/j.neuron.2006.02.016

Wake, H., Lee, P. R., \& Fields, R. D. (2011). Control of local protein synthesis and initial events in myelination by action potentials. Science, 333(6049), 1647-1651. https://doi.org/10.1126/science.1206998

Wake, H., Ortiz, F. C., Woo, D. H., Lee, P. R., Angulo, M. C., \& Fields, R. D. (2015). Nonsynaptic junctions on myelinating glia promote preferential myelination of electrically active axons. Nature Communications, 6, 7844. https://doi.org/10.1038/ncomms8844

Walhovd, K. B., Johansen-Berg, H., \& Káradóttir, R. T. (2014). Unraveling the secrets of white matter-bridging the gap between cellular, animal and human imaging studies. Neuroscience, 276, 2-13. https://doi.org/10. 1016/j.neuroscience.2014.06.058

Wang, F., Ren, S.-Y., Chen, J.-F., Liu, K., Li, R.-X., Li, Z.-F., Hu, B.o, Niu, J.-Q. Xiao, L., Chan, J. R., \& Mei, F. (2020). Myelin degeneration and diminished myelin renewal contribute to age-related deficits in memory. Nature Neuroscience, 23(4), 481-486. https://doi.org/10.1038/s41593-0200588-8

Wark, B., Lundstrom, B. N., \& Fairhall, A. (2007). Sensory adaptation. Current Opinion in Neurobiology, 17(4), 423-429. https://doi.org/10.1016/j.conb. 2007.07.001

Wilson, M. A. (1971). Optic nerve fibre counts and retinal ganglion cell counts during development of Xenopus laevis (Daudin). Quarterly Journal of Experimental Physiology and Cognate Medical Sciences, 56(2), 83-91. https://doi.org/10.1113/expphysiol.1971.sp002110

Xiao, L., Ohayon, D., Mckenzie, I. A., Sinclair-Wilson, A., Wright, J. L., Fudge, A. D., Emery, B., Li, H., \& Richardson, W. D. (2016). Rapid production of new oligodendrocytes is required in the earliest stages of motorskill learning. Nature Neuroscience, 19(9), 1210-1217. https://doi.org/10. 1038/nn.4351

Xin, W., \& Chan, J. R. (2020). Myelin plasticity: Sculpting circuits in learning and memory. Nature Reviews Neuroscience, 21(12), 682-694. https://doi. org/10.1038/s41583-020-00379-8

Yang, S. M., Michel, K., Jokhi, V., Nedivi, E., \& Arlotta, P. (2020). Neuron classspecific responses govern adaptive myelin remodeling in the neocortex. Science, 370(6523). https://doi.org/10.1126/science.abd2109

Yoshida, M. (1997). Oligodendrocyte maturation in Xenopus laevis. Journal of Neuroscience Research, 50(2), 169-176. https://doi.org/10.1002/(SICI) 1097-4547(19971015)50:2〈169::AID-JNR6〉3.0.CO;2-D

Zatorre, R. J., Fields, R. D., \& Johansen-Berg, H. (2012). Plasticity in gray and white: Neuroimaging changes in brain structure during learning. Nature Neuroscience, 15(4), 528-536. https://doi.org/10.1038/nn.3045

Zhang, L. I., Tao, H. W., Holt, C. E., Harris, W. A., \& Poo, M.-M. (1998). A critical window for cooperation and competition among developing retinotectal synapses. Nature, 395(6697), 37-44. https://doi.org/10.1038/25665

Zhang, L. I., Tao, H.-Z. W., \& Poo, M.-M. (2000). Visual input induces longterm potentiation of developing retinotectal synapses. Nature Neuroscience, 3(7), 708-715. https://doi.org/10.1038/76665

Ziskin, J. L., Nishiyama, A., Rubio, M., Fukaya, M., \& Bergles, D. E. (2007) Vesicular release of glutamate from unmyelinated axons in white matter. Nature Neuroscience, 10(3), 321-330. https://doi.org/10.1038/nn1854

How to cite this article: Chorghay, Z., MacFarquhar, D., Li, V. J., Aufmkolk, S., Schohl, A., Wiseman, P. W., Káradóttir, R. T., \& Ruthazer, E. S. (2021). Activity-dependent alteration of early myelin ensheathment in a developing sensory circuit. Journal of Comparative Neurology, 1-15.

https://doi.org/10.1002/cne.25253 